\title{
Deterministic Relative Attitude Determination of Three-Vehicle Formations
}

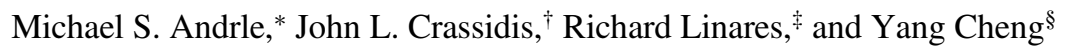 \\ University at Buffalo, State University of New York, Amherst, New York 14260-4400 \\ and \\ Baro Hyun 1 \\ University of Michigan, Ann Arbor, Michigan 48109-2140

\section{DOI: $10.2514 / 1.42849$}

\begin{abstract}
This paper proves that deterministic relative attitude determination is possible for a formation of three vehicles. The results provide an assessment of the accuracy of the deterministic attitude solutions, given statistical properties of the assumed noisy measurements. Each vehicle is assumed to be equipped with sensors to provide line-of-sight, and possibly range, measurements between them. Three vehicles are chosen because this is the minimum number required to determine all attitudes given minimal measurement information. Three cases are studied. The first determines the absolute (inertial) attitude of a vehicle knowing the absolute positions of the other two. The second assumes parallel beams between each vehicle to determine relative attitudes, and the third assumes nonparallel beams for relative attitude determination, which requires range information to find deterministic solutions. Covariance analyses are provided to gain insight on the stochastic properties of the attitude errors and the observability for all three cases.
\end{abstract}

\section{Introduction}

A TTITUDE determination is the calculation of the orientation between two reference frames, two objects, or a reference frame and an object. The amount of research conducted for this task as well as the quantity of related publications is quite extensive, mostly shown in the spacecraft community. For example, a star tracker is used onboard a space vehicle to observe line-of-sight (LOS) vectors to stars, which are compared with known inertial LOS vectors to estimate the inertial attitude of the space vehicle. It is obvious why this topic has acquired so much attention, as nearly every spacecraft ever launched into space requires at least some knowledge of its orientation.

Several sensors can be employed to determine the attitude of a vehicle. Basically, these sensors provide arc length or dihedral-angle information, which can be used for practical purposes to provide entire directions. For example, a star tracker [1] provides a direction, and a Global-Positioning System attitude determination system [2] provides the cosine of an angle. Attitude determination can be broken into two categories: 1) purely deterministic, where a minimal set of data is provided, and 2) overdeterministic, where more than the minimal set is provided. A purely deterministic solution example involves one direction and one angle, essentially giving three equations and three unknowns. A solution for this case is shown in [3]. Two nonparallel directions, such as two LOS vectors to different stars, provide an overdeterministic case, because there are four equations and three unknowns. Solutions to this case generally

Received 19 December 2008; revision received 17 March 2009; accepted for publication 31 March 2009. Copyright $\odot 2009$ by the American Institute of Aeronautics and Astronautics, Inc. All rights reserved. Copies of this paper may be made for personal or internal use, on condition that the copier pay the $\$ 10.00$ per-copy fee to the Copyright Clearance Center, Inc., 222 Rosewood Drive, Danvers, MA 01923; include the code 0731-5090/09 and \$10.00 in correspondence with the CCC.

*Graduate Student, Department of Mechanical \& Aerospace Engineering; msandrle@buffalo.edu.

'Professor, Department of Mechanical \& Aerospace Engineering; johnc@buffalo.edu. Associate Fellow AIAA.

\#Undergraduate Student, Department of Mechanical \& Aerospace Engineering; linares2@buffalo.edu.

${ }^{\S}$ Research Assistant Professor, Department of Mechanical \& Aerospace Engineering; cheng3@buffalo.edu. Senior Member AIAA.

"Graduate Student, Department of Aerospace Engineering; bhyun@ umich.edu. Student Member AIAA. involve solving the classic Wahba problem [4], which has been well studied. A survey of algorithms that solve Wahba's problem is presented in [5].

Formation flying employs multiple vehicles to maintain a specific relative attitude/position, either a statically or a dynamically closed trajectory. Here, relative is defined as being between two vehicles. Relative information is needed to maintain formation attitude through control. Applications are numerous, involving all types of vehicles, including land (robotics [6]), sea (autonomous underwater vehicles [7]), space (spacecraft formations [8]), and air (uninhabited air vehicles [9]) systems. Relative attitude and position estimation schemes based on the Kalman filter have been shown for both spacecraft [10] and aircraft [11] formations. LOS observations are assumed between vehicles based on a system consisting of an optical sensor combined with a specific light source (emitter) to achieve selective vision [12]. Deterministic solutions for both relative attitude and position are possible using multiple emitters [13]. A similar concept has been employed in [14] for robotic pose estimation using multiple LOS observations from image data instead of emitter sources. Other sensors, such as aligned laser communication devices [15], can also be used to provide LOS observations. The use of laser communication devices has increased in recent years. The approach shown in this paper can use these alreadyemployed devices to determine relative attitude information without the use of other (external) attitude sensors, such as star trackers.

In the aforementioned applications, multiple LOS vectors are used to determine relative attitude between vehicles. For example, consider a two-vehicle system with multiple emitters on the deputy vehicle and a focal-plane detector (FPD) on the chief vehicle. For the sake of simplicity, let us assume that the relative position is known. Because the emitter location is known with respect to the deputy frame, then a corresponding reference deputy-frame vector is given. Using the LOS observation from the FPD gives a vector with respect to the chief frame. These vectors are related through the attitude matrix. It is well known that using only one emitter source provides only two of the three pieces of needed attitude information. Hence, multiple LOS observations from multiple emitter sources must be employed to determine a full attitude solution. This is related to the classic photogrammetry problem [16]. The key contribution of this paper is to show that a relative attitude solution can be obtained using single-LOS observations between two pairs of vehicles but employing a three-vehicle formation system. A relative attitude 
solution is not possible if only each vehicle pair in the formation is considered separately. But when all three vehicle LOS observations are considered together, then a purely deterministic solution is possible, which is shown here.

The organization of this paper is as follows. First, the problem definition and notation are stated for the three-vehicle formation. Then the sensor model for the LOS measurements is reviewed. Next, three attitude determination cases are shown: 1) an inertial attitude case, 2) a parallel-beam case for relative attitude determination, and 3) a nonparallel-beam case for relative attitude determination. Covariance expressions are also derived for all three cases. Finally, simulation results are shown.

\section{Problem Definition}

The geometry of the problem is described in Fig. 1. There are three vehicles flying in formation, each vehicle equipped with optical-type sensors, such as a beacon or laser communication system, that use a FPD. Through the sensor, a vehicle measures the LOS vector to the two other vehicles, and this applies to each vehicle, making three pairs of LOS measurements. All LOS measurements from the different vehicles contain noise, even for measurements of the same LOS vector using a different reference frame. The attitude solutions are obtained using a centralized approach in the sense that information from all vehicles is assumed to be transmitted to one location. Decentralized attitude estimation is discussed in [17]. Also, it is assumed that no communication delays are present in the system. A discussion and a mitigation approach for communication delays is shown in [18].

Both theoretical research and supporting simulations will require LOS vectors that describe, with respect to one object or frame, what direction another given object is along. Because different reference frames are used to represent the various LOS vectors, a structured notation is required here as well. A subscript will describe the vehicle for which the LOS is taken both from and to, and a superscript will denote to which reference frame the LOS is both represented by and measured in. For example, $\mathbf{b}_{x / y}^{x}=-\mathbf{b}_{y / x}^{x}$ is a LOS vector beginning at $x$ (using an emitter) and ending at $y$ (using a detector), and it is both expressed in and observed from frame $\mathcal{X}$. Note that from Fig. 1, the detectors measure all vectors that point into the vehicles, and the emitters are used to generate vectors that point away from the vehicles. Now the LOS vectors in Fig. 1 are properly defined.

For the relative attitude matrix, the notation $A_{x}^{y}$ denotes the attitude matrix that maps components expressed in $\mathcal{X}$-frame coordinates to components expressed in $\mathcal{Y}$-frame coordinates, and so $\mathbf{b}_{x / y}^{x}=$ $A_{y}^{x} \mathbf{b}_{x / y}^{y}$. The inverse operator is simply $A_{x}^{y^{T}} \equiv A_{y}^{x}$. There are only two relative attitudes to be determined for the three-vehicle system. Using the characteristic of the attitude matrix, the third attitude is easily obtainable if two relative attitudes are given. For example, knowing $A_{c}^{d_{1}}$ and $A_{c}^{d_{2}}$ gives $A_{d_{1}}^{d_{2}}=A_{c}^{d_{2}} A_{c}^{d_{1}^{T}}$. Using the configuration of the LOS vector measurements between vehicles, this paper will prove that a deterministic solution for the relative attitude can be found. More detailed literature about deterministic attitude determination can be found in [3].

Three cases will be shown in this paper:

1) In the inertial attitude case, the two deputies are treated as reference points and the inertial positions of each vehicle are assumed to be known. For this case, only LOS vectors from the chief to each deputy are required (not between the two deputies) and the determined attitude is with respect to an inertial frame.

2) In the parallel-beam case, the beams between vehicles are assumed to be parallel, and so common vectors are given between vehicles but in different coordinates. For example, for a laser communication system, a feedback device can be employed to ensure that parallel beams are given in real time. As long as the communication system latencies are sufficiently known and the link distance divided by the speed of light is greater than the latencies, the communication system can simply be used as a repeater (or relay if the signal strength is sufficient). It will be shown that deterministic solutions for all relative attitudes with three vehicles are possible for the parallel-beam case.

3) In the nonparallel-beam case, it is assumed that nonparallel beams are present. To achieve common vectors, additional knowledge of range information is required in this case. The attitude solutions are identical to the parallel-beam case; however, additional attitude errors are introduced as a result of the range measurements.

Although the unknown relative attitude is deterministically solvable, LOS vector measurements are usually associated with measurement errors. Therefore, it is critical to investigate the confidence of the attitude solution that is given deterministically with respect to the amount of error that is involved in the LOS measurement. A covariance analysis gives an analytical interpretation regarding this issue. Before showing this analysis, though, we begin with the sensor model used for the LOS measurements.

\section{Sensor Model}

A FPD sensor is assumed for all LOS observations, where $\alpha$ and $\beta$ are the image-space LOS observations. Denoting $\alpha$ and $\beta$ by the $2 \times 1$ vector $\mathbf{m} \equiv\left[\begin{array}{ll}\alpha & \beta\end{array}\right]^{T}$, the measurement model follows:

$$
\tilde{\mathbf{m}}=\mathbf{m}+\mathbf{w}
$$

where $\tilde{\mathbf{m}}$ denotes measurement. A typical noise model used to describe the uncertainty in the focal-plane coordinate observations is given as [10]

$$
\begin{gathered}
\mathbf{w} \sim \mathcal{N}\left(\mathbf{0}, R^{\text {focal }}\right) \\
R^{\text {focal }}=\frac{\sigma^{2}}{1+d\left(\alpha^{2}+\beta^{2}\right)}\left[\begin{array}{cc}
\left(1+d \alpha^{2}\right)^{2} & (d \alpha \beta)^{2} \\
(d \alpha \beta)^{2} & \left(1+d \beta^{2}\right)^{2}
\end{array}\right]
\end{gathered}
$$

where $\sigma^{2}$ is the variance of the measurement errors associated with $\alpha$ and $\beta$, and $d$ is on the order of 1 .

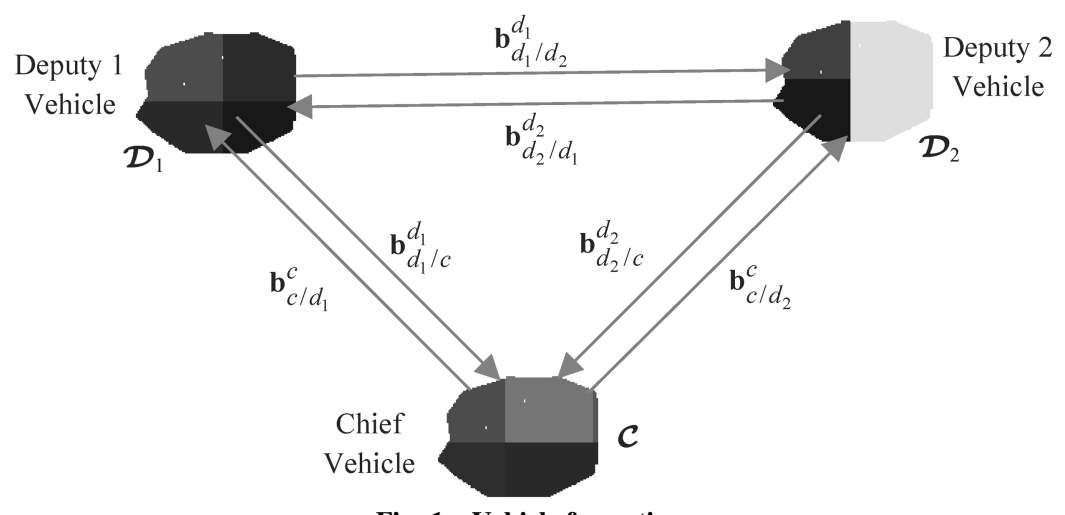

Fig. 1 Vehicle formation. 
Assuming a focal length of unity, the sensor LOS observations can be expressed in unit vector form, which is given by

$$
\mathbf{b}=\frac{1}{\sqrt{1+\alpha^{2}+\beta^{2}}}\left[\begin{array}{c}
\alpha \\
\beta \\
1
\end{array}\right]
$$

The measurement vector is defined as

$$
\tilde{\mathbf{b}}=\mathbf{b}+\boldsymbol{v}
$$

with

$$
v \sim \mathcal{N}(\mathbf{0}, \Omega)
$$

Under the assumption that the focal-plane measurements are normally distributed with known mean and covariance, it is further assumed that under the focal-plane-to-LOS transformation, the resulting LOS uncertainty is approximately Gaussian. Also recall that because a LOS vector is of unit length it must lie on the unit sphere, which leads to a rank-deficient covariance matrix in $\mathcal{R}^{3}$. To characterize the LOS noise process resulting from the focal-plane model, Shuster [19] suggests the following approximation:

$$
\Omega \equiv E\left\{\boldsymbol{v} \boldsymbol{v}^{T}\right\}=\sigma^{2}\left(I_{3 \times 3}-\mathbf{b b}^{T}\right)
$$

known as the QUEST measurement model (QMM). A geometric interpretation of the covariance given by the QMM can be obtained by first considering the outer product. The operator formed by the outer product of a vector, $\mathbf{b}$ with itself, is a projection operator for which the image is the component of the domain spanned by $\mathbf{b}$. Similarly, the operator $\left(I_{3 \times 3}-\mathbf{b b}^{T}\right)$ is also a projection, this time yielding an image perpendicular to $\mathbf{b}$.

What this means for the covariance given in Eq. (6) is that the error in the vector $\tilde{\mathbf{b}}$ is assumed to lie in a plane tangent to the focal sphere. It is clear that this is only valid for small $\alpha$ and $\beta$ values in which a tangent plane closely approximates the surface of a unit sphere. For small $\alpha$ and $\beta$ values, the QMM model agrees well with the inferred measurement model for the real sensor though [19]. For wide-fieldof-view (WFOV) sensors, which may produce large $\alpha$ and $\beta$ values, a more accurate measurement covariance is shown in [20]. This formulation employs a first-order Taylor series approximation about the focal-plane axes. The partial derivative operator is used to linearly expand the focal-plane covariance in Eq. (2), given by

$$
H=\frac{\partial \mathbf{b}}{\partial \mathbf{m}}=\frac{1}{\sqrt{1+\alpha^{2}+\beta^{2}}}\left[\begin{array}{ll}
1 & 0 \\
0 & 1 \\
0 & 0
\end{array}\right]-\frac{1}{1+\alpha^{2}+\beta^{2}} \mathbf{b m}^{T}
$$

Then the WFOV covariance model is given by

$$
\Omega=H R^{\text {focal }} H^{T}
$$

If a small-field-of-view model is valid, then Eq. (ㅇ) can still be used but is nearly identical to Eq. (6). For both equations, $\Omega$ is a singular matrix [20]. The implications of this singularity will be discussed later. Also note that from Eq. (8), different body-frame vectors $\mathbf{b}$ give different corresponding covariance matrices. Hence, from this point forward, the notation will specifically show the frames used for both the body vector and its associated covariance. In particular, the six body-vector measurements from the onboard sensors, along with their respective error characteristics, are given by

$$
\begin{aligned}
\tilde{\mathbf{b}}_{d_{2} / d_{1}}^{d_{2}}=\mathbf{b}_{d_{2} / d_{1}}^{d_{2}}+\boldsymbol{v}_{d_{2} / d_{1}}^{d_{2}}, & \boldsymbol{v}_{d_{2} / d_{1}}^{d_{2}} \sim \mathcal{N}\left(\mathbf{0}, \Omega_{d_{2} / d_{1}}^{d_{2}}\right) \\
\tilde{\mathbf{b}}_{d_{2} / d_{1}}^{d_{1}}=\mathbf{b}_{d_{2} / d_{1}}^{d_{1}}+\boldsymbol{v}_{d_{2} / d_{1}}^{d_{1}}, & \boldsymbol{v}_{d_{2} / d_{1}}^{d_{1}} \sim \mathcal{N}\left(\mathbf{0}, \Omega_{d_{2} / d_{1}}^{d_{1}}\right) \\
\tilde{\mathbf{b}}_{c / d_{1}}^{c}=\mathbf{b}_{c / d_{1}}^{c}+\boldsymbol{v}_{c / d_{1}}^{c}, & \boldsymbol{v}_{c / d_{1}}^{c} \sim \mathcal{N}\left(\mathbf{0}, \Omega_{c / d_{1}}^{c}\right) \\
\tilde{\mathbf{b}}_{c / d_{1}}^{d_{1}}=\mathbf{b}_{c / d_{1}}^{d_{1}}+\boldsymbol{v}_{c / d_{1}}^{d_{1}}, & \boldsymbol{v}_{c / d_{1}}^{d_{1}} \sim \mathcal{N}\left(\mathbf{0}, \Omega_{c / d_{1}}^{d_{1}}\right)
\end{aligned}
$$

$$
\begin{array}{rlrl}
\tilde{\mathbf{b}}_{c / d_{2}}^{c}=\mathbf{b}_{c / d_{2}}^{c}+\boldsymbol{v}_{c / d_{2}}^{c}, & & \boldsymbol{v}_{c / d_{2}}^{c} \sim \mathcal{N}\left(\mathbf{0}, \Omega_{c / d_{2}}^{c}\right) \\
\tilde{\mathbf{b}}_{c / d_{2}}^{d_{2}}=\mathbf{b}_{c / d_{2}}^{d_{2}}+\boldsymbol{v}_{c / d_{2}}^{d_{2}}, & \boldsymbol{v}_{c / d_{2}}^{d_{2}} \sim \mathcal{N}\left(\mathbf{0}, \Omega_{c / d_{2}}^{d_{2}}\right)
\end{array}
$$

Note that all noise terms in Eq. (9) are assumed to be uncorrelated.

\section{Inertial Attitude Case}

The problem to be solved here is as follows: given relative LOS vectors and inertial positions of each vehicle, determine the inertial attitude of one vehicle at a time. Once the inertial attitudes are determined, then converting them to relative attitudes is straightforward. This case assumes a three-vehicle configuration in which each vehicle communicates with its two neighbors. Using only relative LOS observations between each vehicle does not allow for a deterministic inertial attitude solution in this case. Hence, more information must be employed. Here, it is assumed that the absolute position of each vehicle is known. Figure 2 shows the inertial position vectors for a chief and deputy case, in which the superscript $i$ denotes inertial coordinates and $\mathbf{p}_{c / d_{1}}^{i}=\mathbf{p}_{d_{1}}^{i}-\mathbf{p}_{c}^{i}$. The absolute position of a deputy can be determined using relative observations between vehicles and absolute information of one vehicle in the formation. The relative unit vector is given by

$$
\mathbf{r}_{c / d_{1}}^{i}=\mathbf{p}_{c / d_{1}}^{i} /\left\|\mathbf{p}_{c / d_{1}}^{i}\right\|
$$

This vector is observed in the body frame of the vehicle, denoted by $\mathbf{b}_{c / d_{1}}^{c}$. The mapping between the vectors $\mathbf{r}_{c / d_{1}}^{i}$ and $\mathbf{b}_{c / d_{1}}^{c}$ is given by

$$
\mathbf{b}_{c / d_{1}}^{c}=A_{i}^{c} \mathbf{r}_{c / d_{1}}^{i}
$$

where $A_{i}^{c}$ is the attitude matrix. The same mapping can be applied to the chief and second deputy vectors, giving $\mathbf{r}_{c / d_{2}}^{i}$ and $\mathbf{b}_{c / d_{2}}^{c}$ with the same attitude mapping $A_{i}^{c}$.

The main issue here is that noise is present not only in the LOS observations, but also in the position knowledge. Ignoring subscripts and superscripts for the moment, the measurement model follows:

$$
\tilde{\mathbf{b}}=A \tilde{\mathbf{r}}+\boldsymbol{v}
$$

where $\tilde{\mathbf{r}}$ is the measured quantity of $\mathbf{r}$, and $\boldsymbol{v}$ has covariance $\Omega$, which has been previously discussed. If the position error is small, then a first-order expansion of the noise process in $\tilde{\mathbf{r}}$ is possible. The error process for the position vector is given by

$$
\tilde{\mathbf{p}}=\mathbf{p}+\delta \mathbf{p}
$$

where $\delta \mathbf{p}$ is the first-order error term in the position vector. The covariance of $\delta \mathbf{p}$ is denoted by $\Omega_{p}$. Using a Taylor series expansion and neglecting second-order terms, to within first order, $\tilde{\mathbf{r}}$ is approximated by

$$
\tilde{\mathbf{r}}=\mathbf{r}+\delta \mathbf{r}
$$

where the covariance of $\delta \mathbf{r}$ is given by

$$
\Omega_{r}=\left(\frac{\partial \mathbf{r}}{\partial \mathbf{p}}\right) \Omega_{p}\left(\frac{\partial \mathbf{r}}{\partial \mathbf{p}}\right)^{T}
$$

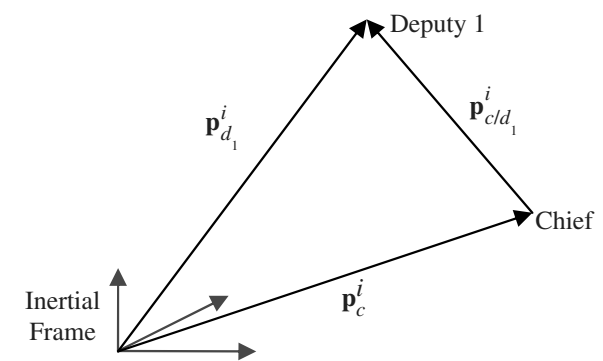

Fig. 2 Inertial position vectors for chief and deputy 1. 
with

$$
\frac{\partial \mathbf{r}}{\partial \mathbf{p}}=\|\mathbf{p}\|^{-1}\left(I_{3 \times 3}-\|\mathbf{p}\|^{-2} \mathbf{p} \mathbf{p}^{T}\right)=-\|\mathbf{p}\|^{-3}[\mathbf{p} \times]^{2}
$$

where $[\mathbf{p} \times]$ is the standard cross-product matrix [21]. Therefore, assuming that $\boldsymbol{v}$ and $\delta \mathbf{r}$ are uncorrelated, the measurement-error covariance for $\boldsymbol{v}$ in Eq. (12) is given by

$$
R=A \Omega_{r} A^{T}+\Omega
$$

Note that $R$ is a function of the unknown attitude matrix. We now prove that this matrix is a singular matrix using the QMM for $\Omega$. Using the identity $A[\mathbf{p} \times]=[A \mathbf{p} \times] A$ (see [21]) and the identity $\mathbf{b}=A \mathbf{p} /\|\mathbf{p}\|$, then the matrix $A \Omega_{r} A^{T}$ can be written as

$$
A \Omega_{r} A^{T}=\|\mathbf{p}\|^{-2}[\mathbf{b} \times]^{2} A \Omega_{p} A^{T}[\mathbf{b} \times]^{2}
$$

It is obvious now that $R \mathbf{b}=\mathbf{0}$, which means that $R$ is singular. This matrix is also singular using the WFOV model, because $\mathbf{b}$ is in the null space of $\Omega$ given by Eq. (8), due to $H^{T} \mathbf{b}=\mathbf{0}$, and is also in the null space of $A \Omega_{r} A^{T}$.

A discussion on a probability density function (pdf) with a singular covariance matrix is now given. This is required later to develop the negative log-likelihood function. Suppose that $\mathbf{x}$ is a zero-mean Gaussian distribution with nonsingular covariance $R_{x}$. Its pdf is given by

$$
p(\mathbf{x})=\frac{1}{\left[\operatorname{det}\left(2 \pi R_{x}\right)\right]^{1 / 2}} \exp \left(-\frac{1}{2} \mathbf{x}^{T} R_{x}^{-1} \mathbf{x}\right)
$$

Let $\mathbf{y}=U \mathbf{x}$, where $U^{T} U=I$ and $U$ maps $\mathbf{x}$ into a higher dimensional space. So $\mathbf{x}=U^{T} \mathbf{y}$, and the singular covariance matrix of $\mathbf{y}$ is given by $R_{y}=U R_{x} U^{T}$. Rewriting $p(\mathbf{x})$ in terms of $\mathbf{y}$ leads to

$$
p(\mathbf{y})=\frac{1}{\left[\operatorname{det}\left(2 \pi U^{T} R_{y} U\right)\right]^{1 / 2}} \exp \left(-\frac{1}{2} \mathbf{y}^{T} R_{y}^{\dagger} \mathbf{y}\right)
$$

where $\mathbf{y}$ is in a subspace in the higher dimensional space defined by the mapping $U, R_{y}^{\dagger}=U R_{x}^{-1} U^{T}$ denotes the pseudoinverse of $R_{y}$, and $U^{T} R_{y} U=R_{x}$. The value of $\operatorname{det}\left(U^{T} R_{y} U\right)=\operatorname{det}\left(R_{x}\right)$ is equal to the product of the nonzero eigenvalues of $R_{y}$. For our work, we have $R_{y} \equiv R$, where $R$ is the singular matrix given by Eq. (17). The singularity of $R$ means that the probability density function is effectively two-dimensional. Instead of including a term $\ln [\operatorname{det}(R)]$, the negative log-likelihood function associated with this singular covariance matrix includes a term $\ln \left[\operatorname{det}\left(U^{T} R U\right)\right]$, with the $3 \times 2$ matrix $U$ satisfying $U U^{T}=I_{3 \times 3}-\mathbf{b b}^{T}$. The $\ln$-det term can be ignored in the log-likelihood function, because $U^{T} R U$ is independent of $\mathbf{b}$ or $A$, which results from our assumption that the noise distribution in the plane perpendicular to $\mathbf{b}$ is independent of $\mathbf{b}$. Note that the null vector of the WFOV model in Eq. (8) is also b [20], and so the same statement applies to this model as well.

The negative log-likelihood function to determine $A_{i}^{c}$ is given by

$$
\begin{gathered}
J\left(A_{i}^{c}\right)=\frac{1}{2}\left(\tilde{\mathbf{b}}_{c / d_{1}}^{c}-A_{i}^{c} \tilde{\mathbf{r}}_{c / d_{1}}^{i}\right)^{T} R_{c / d_{1}}^{-1}\left(\tilde{\mathbf{b}}_{c / d_{1}}^{c}-A_{i}^{c} \tilde{\mathbf{r}}_{c / d_{1}}^{i}\right) \\
+\frac{1}{2}\left(\tilde{\mathbf{b}}_{c / d_{2}}^{c}-A_{i}^{c} \tilde{\mathbf{r}}_{c / d_{2}}^{i}\right)^{T} R_{c / d_{2}}^{-1}\left(\tilde{\mathbf{b}}_{c / d_{2}}^{c}-A_{i}^{c} \tilde{\mathbf{r}}_{c / d_{2}}^{i}\right)
\end{gathered}
$$

with

$$
\begin{aligned}
& R_{c / d_{1}}=A_{i}^{c}\left(\frac{\partial \mathbf{r}_{c / d_{1}}^{i}}{\partial \mathbf{p}_{c / d_{1}}^{i}}\right) \Omega_{p_{1}}\left(\frac{\partial \mathbf{r}_{c / d_{1}}^{i}}{\partial \mathbf{p}_{c / d_{1}}^{i}}\right)^{T} A_{i}^{c^{T}}+\Omega_{c / d_{1}}^{c} \\
& R_{c / d_{2}}=A_{i}^{c}\left(\frac{\partial \mathbf{r}_{c / d_{2}}^{i}}{\partial \mathbf{p}_{c / d_{2}}^{i}}\right) \Omega_{p_{2}}\left(\frac{\partial \mathbf{r}_{c / d_{2}}^{i}}{\partial \mathbf{p}_{c / d_{2}}^{i}}\right)^{T} A_{i}^{c^{T}}+\Omega_{c / d_{2}}^{c}
\end{aligned}
$$

where $\Omega_{p_{1}}$ and $\Omega_{p_{2}}$ are the covariances associated with the errors in $\tilde{\mathbf{p}}_{c / d_{1}}^{i}$ and $\tilde{\mathbf{p}}_{c / d_{2}}^{i}$, respectively. As shown previously, the matrices $R_{c / d_{1}}$ and $R_{c / d_{2}}$ are singular. However, Shuster [22] has shown that these matrices can effectively be replaced with nonsingular matrices, which does not affect the likelihood function. This approach was expanded for wide fields of view in [20]. For example, the matrix $R_{c / d_{1}}$ can be replaced by $R_{c / d_{1}}+\frac{1}{2} \mathbf{b}_{c / d_{1}}^{c} \mathbf{b}_{c / d_{1}}^{c^{T}} \operatorname{tr}\left(R_{c / d_{1}}\right)$, which is a nonsingular matrix. The resulting new matrices are generally not diagonal matrices, and so a standard attitude determination algorithm, such as QUEST [23], cannot be directly applied. A solution can be found by assuming that $R_{c / d_{1}}$ and $R_{c / d_{2}}$ are diagonal, using QUEST to find an approximate solution, which is then used in an iterative least-squares approach to determine the optimal estimate for $A_{i}^{c}$ [24]. Also, the approach in [25] can be used to determine the attitude-error covariance of $A_{i}^{c}$, which can be shown to be given by

$$
\begin{aligned}
P_{c}^{i} & =\left\{\left[\mathbf{b}_{c / d_{1}}^{c} \times\right]\left[R_{c / d_{1}}+\frac{1}{2} \mathbf{b}_{c / d_{1}}^{c} \mathbf{b}_{c / d_{1}}^{c^{T}} \operatorname{tr}\left(R_{c / d_{1}}\right)\right]^{-1}\left[\mathbf{b}_{c / d_{1}}^{c} \times\right]^{T}\right. \\
& +\left[\left[\mathbf{b}_{c / d_{2}}^{c} \times\right]\left[R_{c / d_{2}}+\frac{1}{2} \mathbf{b}_{c / d_{2}}^{c} \mathbf{b}_{c / d_{2}}^{c^{T}} \operatorname{tr}\left(R_{c / d_{2}}\right)\right]^{-1}\left[\mathbf{b}_{c / d_{2}}^{c} \times\right]^{T}\right\}^{-1}
\end{aligned}
$$

Note that $P_{c}^{i}$ is evaluated using the true values, but the measured or estimated ones can be used, which leads to only second-order effects [25].

A simulation is now shown that assesses how the position errors affect the overall covariance given in Eq. (22). In particular, only the first term on the right-hand side of Eq. (22) is investigated; the second term is independent of position error and position. A two-spacecraft configuration is used with relative positions starting at low Earth orbit $(300 \mathrm{~km})$ up to geostationary orbits $(42,164 \mathrm{~km})$ separated by $2 \mathrm{deg}$. The attitude matrix of the chief is assumed to be the identity matrix. The position-error covariance is assumed to be isotropic (a scalar times identity matrix), with $3 \sigma$ bounds for the position errors ranging from 0.1 to $100 \mathrm{~km}$. The average $3 \sigma$ bounds for the noise induced by the position errors are shown in Fig. 3. Clearly, the position errors can provide significant error effects into the overall process if precise attitude knowledge is required.

\section{Parallel-Beam Case}

The problem to be solved here is as follows: given relative and parallel LOS vector pairs, determine the relative attitudes between each vehicle. The LOS measurement equations for each vehicle pair are given by

$$
\begin{gathered}
\tilde{\mathbf{b}}_{c / d_{1}}^{c}=A_{d_{1}}^{c} \tilde{\mathbf{b}}_{c / d_{1}}^{d_{1}} \\
\tilde{\mathbf{b}}_{c / d_{2}}^{c}=A_{d_{2}}^{c} \tilde{\mathbf{b}}_{c / d_{2}}^{d_{2}} \\
\tilde{\mathbf{b}}_{d_{2} / d_{1}}^{d_{2}}=A_{c}^{d_{2}} A_{d_{1}}^{c} \tilde{\mathbf{b}}_{d_{2} / d_{1}}^{d_{1}}=A_{d_{1}}^{d_{2}} \tilde{\mathbf{b}}_{d_{2} / d_{1}}^{d_{1}}
\end{gathered}
$$

The model in Eq. (24) assumes parallel-beams, which must be maintained through hardware calibrations. Taking the dot product of Eqs. (24a) and (24b) gives

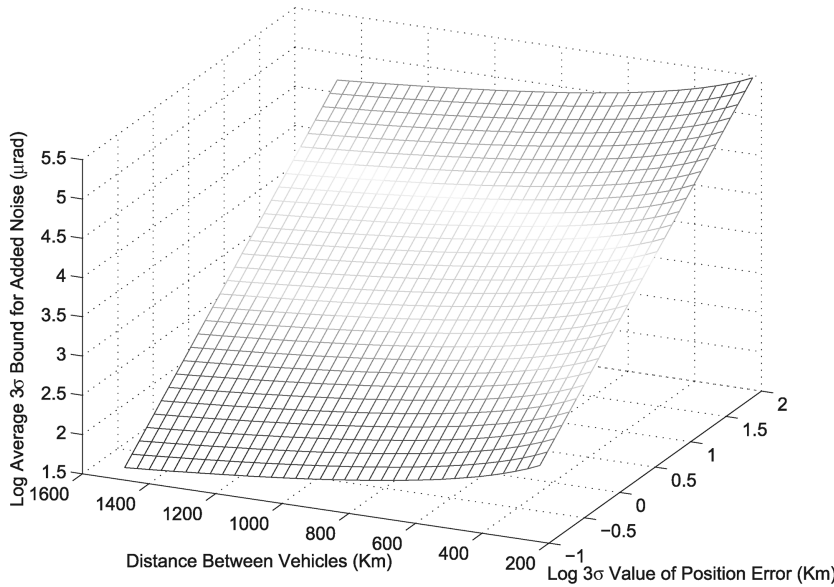

Fig. 3 Average $3 \sigma$ bounds. 


$$
\tilde{\mathbf{b}}_{c / d_{2}}^{c^{T}} \tilde{\mathbf{b}}_{c / d_{1}}^{c}=\tilde{\mathbf{b}}_{c / d_{2}}^{d_{2}^{T}} A_{d_{1}}^{d_{2}} \tilde{\mathbf{b}}_{c / d_{1}}^{d_{1}}
$$

Equations (24c) and (25) represent a direction and an angle, respectively, which can be used to determine $A_{d_{1}}^{d_{2}}$, given by an algorithm in [3]. This algorithm is now reviewed. Considering the measurements shown in Fig. 4, to determine the full attitude between the $\mathcal{D}_{2}$ and $\mathcal{D}_{1}$ frames, we must find the attitude matrix that satisfies the following general relations:

$$
\begin{aligned}
& \mathbf{w}_{1}=A \mathbf{v}_{1} \\
& d=\mathbf{s}^{T} A \mathbf{v}_{2}
\end{aligned}
$$

where $d$ and all vectors in Eq. (26) are given. Also, all vectors have unit length. The solution can be found by first finding an attitude matrix that satisfies Eq. (26a) and then finding the angle that one must rotate about the reference direction to satisfy Eq. (26b). The first rotation can be found by rotating about any direction by an angle $\theta$, with $B=R\left(\mathbf{n}_{1}, \theta\right)$, where $R\left(\mathbf{n}_{1}, \theta\right)$ is a general rotation about some rotational axis $\mathbf{n}_{1}$ that satisfies $\mathbf{w}_{1}=B \mathbf{v}_{1}$. The choice of the initial rotation axis is arbitrary; here, the vector between the two referencedirection vectors is used, and so

$$
B=\frac{\left(\mathbf{v}_{1}+\mathbf{w}_{1}\right)\left(\mathbf{v}_{1}+\mathbf{w}_{1}\right)^{T}}{\left(1+\mathbf{v}_{1}^{T} \mathbf{w}_{1}\right)}-I_{3 \times 3}
$$

where $\mathbf{n}_{1}=\mathbf{v}_{1}+\mathbf{w}_{1}$. The vector $\mathbf{w}^{*}$ is now defined, which is the vector produced after applying the rotation $B$ on the vector $\mathbf{v}_{2}$. This will allow us to determine the second rotation needed to map $\mathbf{v}_{2}$ to the second frame: $\mathbf{w}^{*}=B \mathbf{v}_{2}$. Because the rotation axis is about the $\mathbf{w}_{1}$ vector, this vector will be invariant under this transformation, and the solution to the full attitude can be written as $A=R\left(\mathbf{n}_{2}, \theta\right) B$. A rotation that satisfies the following equation must be found: $d=\mathbf{s}^{T} R\left(\mathbf{n}_{2}, \theta\right) \mathbf{w}^{*}$, where

$$
R\left(\mathbf{n}_{2}, \theta\right)=\cos (\theta) I_{3 \times 3}+[1-\cos (\theta)] \mathbf{n}_{2} \mathbf{n}_{2}^{T}-\sin (\theta)\left[\mathbf{n}_{2} \times\right]
$$

Substituting Eq. (28) with $\mathbf{n}_{2}=\mathbf{w}_{1}$ into $d$ and then rearranging terms leads to

$$
\begin{gathered}
\left\|\mathbf{s} \times \mathbf{w}_{1}\right\|\left\|\mathbf{w}_{1} \times \mathbf{w}^{*}\right\| \cos (\theta-\varphi)=\left(\mathbf{s}^{T} \mathbf{w}_{1}\right)\left(\mathbf{w}_{1}^{T} \mathbf{w}^{*}\right)-d \\
\varphi=\operatorname{atan}_{2}\left[\mathbf{s}^{T}\left(\mathbf{w}_{1} \times \mathbf{w}^{*}\right), \mathbf{s}^{T}\left(\mathbf{w}_{1} \times\left(\mathbf{w}_{1} \times \mathbf{w}^{*}\right)\right)\right]
\end{gathered}
$$

Then the angle for the rotation about $\mathbf{w}_{1}$ is

$$
\theta=\varphi+\cos ^{-1}\left[\frac{\left(\mathbf{s}^{T} \mathbf{w}_{1}\right)\left(\mathbf{w}_{1}^{T} \mathbf{w}^{*}\right)-d}{\left\|\mathbf{s} \times \mathbf{w}_{1}\right\|\left\|\mathbf{w}_{1} \times \mathbf{w}^{*}\right\|}\right]
$$

The inverse cosine function returns the same solution for angles in the first and forth quadrants and for angles in the second and third quadrants. This will create a twofold ambiguity, which is easily resolved from the geometry of the vehicle system. For example, once an attitude solution has been determined, then it can be used to compute estimated body vectors, which can be checked to see whether or not they are within the field of view of the sensor. The argument of this function cannot be greater than one, and so the following inequality must be satisfied for a solution to exist:

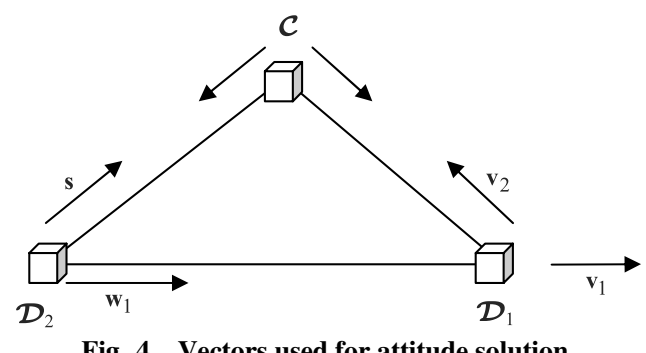

Fig. 4 Vectors used for attitude solution.

$$
\left|\left(\mathbf{s}^{T} \mathbf{w}_{1}\right)\left(\mathbf{w}_{1}^{T} \mathbf{w}^{*}\right)-d\right| \leq\left\|\mathbf{s} \times \mathbf{w}_{1}\right\|\left\|\mathbf{w}_{1} \times \mathbf{w}^{*}\right\|
$$

With this attitude determination method, there are some cases in which a solution does not exist. It will be shown later that planar vectors yield an unobservable system.

The attitude solution is given by

$$
A=R\left(\mathbf{w}_{1}, \theta\right) B
$$

For example, to determine $A_{d_{1}}^{d_{2}}$, choose $d=\tilde{\mathbf{b}}_{c / d_{2}}^{c^{T}} \tilde{\mathbf{b}}_{c / d_{1}}^{c}, \mathbf{w}_{1}=\tilde{\mathbf{b}}_{d_{2} / d_{1}}^{d_{2}}$, $\mathbf{v}_{1}=\tilde{\mathbf{b}}_{d_{2} / d_{1}}^{d_{1}}, \mathbf{s}=\tilde{\mathbf{b}}_{c / d_{2}}^{d_{2}}$, and $\mathbf{v}_{2}=\tilde{\mathbf{b}}_{c / d_{1}}^{d_{1}}$. The first step is to determine the matrix $B$ in Eq. (27). Then the rotation angle is determined using Eq. (30), followed by determination of the matrix $R\left(\mathbf{n}_{2}, \theta\right)$ using Eq. (28). Finally, the attitude is determined using Eq. (32). It is important to note that without the resolution of the attitude ambiguity, any covariance developed would have no meaning. If the wrong attitude is used, then the errors may be fairly large and not bounded by the attitude-error covariance.

The same procedure can be used to determine the remaining attitudes; however, once the first relative attitude is determined, a standard and computationally efficient attitude determination approach is employed instead. To determine one of the remaining attitudes, the TRIAD algorithm can be employed:

$$
\begin{gathered}
A=M_{c} M_{d}^{T} \\
M_{c}=\left[\begin{array}{lll}
\mathbf{c}_{1} & \frac{\mathbf{c}_{1} \times \mathbf{c}_{2}}{\left\|\mathbf{c}_{1} \times \mathbf{c}_{2}\right\|} & \frac{\mathbf{c}_{1} \times\left(\mathbf{c}_{1} \times \mathbf{c}_{2}\right)}{\left\|\mathbf{c}_{1} \times\left(\mathbf{c}_{1} \times \mathbf{c}_{2}\right)\right\|}
\end{array}\right] \\
M_{d}=\left[\begin{array}{lll}
\mathbf{d}_{1} & \frac{\mathbf{d}_{1} \times \mathbf{d}_{2}}{\left\|\mathbf{d}_{1} \times \mathbf{d}_{2}\right\|} & \frac{\mathbf{d}_{1} \times\left(\mathbf{d}_{1} \times \mathbf{d}_{2}\right)}{\left\|\mathbf{d}_{1} \times\left(\mathbf{d}_{1} \times \mathbf{d}_{2}\right)\right\|}
\end{array}\right]
\end{gathered}
$$

For example, to find $A_{d_{1}}^{c}$, choose $\mathbf{c}_{1}=\tilde{\mathbf{b}}_{c / d_{1}}^{c}, \mathbf{c}_{2}=\tilde{\mathbf{b}}_{c / d_{2}}^{c}, \mathbf{d}_{1}=\tilde{\mathbf{b}}_{c / d_{1}}^{d_{1}}$, and $\mathbf{d}_{2}=A_{d_{2}}^{d_{1}} \tilde{\mathbf{b}}_{c / d_{2}}^{d_{2}}$. Once this attitude is found, the final relative attitude can be determined by simply using $A_{d_{2}}^{c}=A_{d_{1}}^{c} A_{d_{2}}^{d_{1}}$.

\section{A. Covariance Analysis}

To determine the covariance of the attitude error for $A_{d_{1}}^{d_{2}}$, the covariance of the LOS measurement vector in Eq. (24c), and the variance of the dot product in Eq. (25) must be determined, because the solution uses these equations. The attitude-error covariance will be derived using a log-likelihood approach that involves these quantities.

\section{Vector LOS Covariance}

Note that Eq. $(24 \mathrm{c})$ has two noise terms: one associated with $\tilde{\mathbf{b}}_{d_{2} / d_{1}}^{d_{1}}$ and one associated with $\tilde{\mathbf{b}}_{d_{2} / d_{1}}^{d_{2}}$. To determine the overall measurement-error covariance, which is a combination of both terms, the following vector is first defined:

$$
\tilde{\zeta}_{d_{2} / d_{1}}^{d_{2}} \equiv A_{d_{1}}^{d_{2}} \tilde{\mathbf{b}}_{d_{2} / d_{1}}^{d_{1}}-\tilde{\mathbf{b}}_{d_{2} / d_{1}}^{d_{2}}
$$

Substituting Eq. ( $\underline{9})$ into $\tilde{\zeta}_{d_{2} / d_{1}}^{d_{2}}$ leads to

$$
\tilde{\zeta}_{d_{2} / d_{1}}^{d_{2}}=A_{d_{1}}^{d_{2}} \boldsymbol{v}_{d_{2} / d_{1}}^{d_{1}}-\boldsymbol{v}_{d_{2} / d_{1}}^{d_{2}}
$$

Equation ( $\underline{36})$ is linear in the noise terms $\boldsymbol{v}$, and as a result, $\tilde{\zeta}_{d_{2} / d_{1}}^{d_{2}}$ has Gaussian distributed uncertainty that can be described by two parameters: the mean and covariance $\mu_{d_{2} / d_{1}}^{d_{2}}$ and $R_{d_{2} / d_{1}}^{d_{2}}$, respectively:

$$
\begin{gathered}
\boldsymbol{\mu}_{d_{2} / d_{1}}^{d_{2}}=E\left\{\tilde{\zeta}_{d_{2} / d_{1}}^{d_{2}}\right\}=\mathbf{0} \\
R_{d_{2} / d_{1}}^{d_{2}}=E\left\{\left(\tilde{\zeta}_{d_{2} / d_{1}}^{d_{2}}-\mu_{d_{2} / d_{1}}^{d_{2}}\right)\left(\tilde{\zeta}_{d_{2} / d_{1}}^{d_{2}}-\mu_{d_{2} / d_{1}}^{d_{2}}\right)^{T}\right\}
\end{gathered}
$$

Substituting Eqs. (하) and (37) into Eq. (하) and expanding leads to the following expression: 


$$
\begin{gathered}
R_{d_{2} / d_{1}}^{d_{2}}=E\left\{A_{d_{1}}^{d_{2}} \boldsymbol{v}_{d_{2} / d_{1}}^{d_{1}} \boldsymbol{v}_{d_{2} / d_{1}}^{d_{1}^{T}} A_{d_{1}}^{d_{2}^{T}}-A_{d_{1}}^{d_{2}} \boldsymbol{v}_{d_{2} / d_{1}}^{d_{1}} \boldsymbol{v}_{d_{2} / d_{1}}^{d^{T}}\right. \\
\left.-\boldsymbol{v}_{d_{2} / d_{1}}^{d_{2}} \boldsymbol{v}_{d_{2} / d_{1}}^{d_{1}^{T}} A_{d_{1}}^{d_{2}^{T}}+\boldsymbol{v}_{d_{2} / d_{1}}^{d_{2}} \boldsymbol{v}_{d_{2} / d_{1}}^{d^{T}}\right\}
\end{gathered}
$$

Completing the term-by-term expectation in Eq. (39) leads immediately to the measurement covariance expression for the vector LOS:

$$
R_{d_{2} / d_{1}}^{d_{2}}=A_{d_{1}}^{d_{2}} \Omega_{d_{2} / d_{1}}^{d_{1}} A_{d_{1}}^{d_{2}^{T}}+\Omega_{d_{2} / d_{1}}^{d_{2}}
$$

This covariance is a function of the true (and not known) attitude matrix as well as the assumed known noise process characteristics of the vehicle sensors. The two-term solution in Eq. (40) is indicative of the fact that both the measured LOS and the reference vector contain uncertainty. The covariance associated with $\tilde{\mathbf{b}}_{d_{2} / d_{1}}^{d_{1}}$ needs to be transformed to the $\mathcal{D}_{2}$ coordinate space before being summed with the covariance of $\tilde{\mathbf{b}}_{d_{2} / d_{1}}^{d_{2}}$.

There are two primary approaches to address the fact that the covariance is a function of the unknown true relative attitude matrix. First, the true attitude matrix can be approximated by the estimated attitude matrix. This simply requires that the true attitude matrix be replaced by its estimate in all covariance expressions. This method is a good approximation that produces second-order error effects that can be ignored [25]. Second, because each pair of LOS vectors is parallel, the focal planes for each of the two involved sensors are aligned. The body vectors from each emitter/receiver pair are the same once the coordinate transformation is made of the respective covariance, as seen by Eq. (6). Under the logical assumption that both sensors have the same noise characteristics, we have $A_{d_{1}}^{d_{2}} \Omega_{d_{2} / d_{1}}^{d_{1}} A_{d_{1}}^{d_{2}^{T}}=\Omega_{d_{2} / d_{1}}^{d_{2}}$. Making this substitution into Eq. ( $\underline{40)}$ leads to the attitude-independent expression for the covariance, namely,

$$
R_{d_{2} / d_{1}}^{d_{2}}=2 \Omega_{d_{2} / d_{1}}^{d_{2}}
$$

This relation is clearly obvious using the QMM. For the WFOV model, Eq. (41) is only approximately correct. The eigenvectors of both the QMM and the WFOV model are identical; the only difference is in their nonzero eigenvalues [20]. The nonzero eigenvalues of the QMM are both given by $\overline{\sigma^{2}}$. If the nonzero eigenvalues of the WFOV model are close to $\sigma^{2}$, then Eq. (41) is a approximately valid. This can easily be checked using the available measurements. Also, because a purely deterministic solution is possible with a three-vehicle formation, then the covariance of the measurement errors does not affect the attitude solution. That is, there are exactly the same number of equations as unknowns to find a solution, and any weighting of the measurements does not change the solution. Hence, Eq. (41) is only needed to study the bounds on the expected measurement errors, which may be used to perform an initial assessment, using Eq. (40) to determine a more accurate one if needed.

\section{Angle Cosine Variance}

Substituting Eq. (9) into Eq. (25) leads to

$$
\begin{aligned}
& \left(\mathbf{b}_{c / d_{2}}^{c}+\boldsymbol{v}_{c / d_{2}}^{c}\right)^{T}\left(\mathbf{b}_{c / d_{1}}^{c}+\boldsymbol{v}_{c / d_{1}}^{c}\right) \\
& \quad=\left(\mathbf{b}_{c / d_{2}}^{d_{2}}+\boldsymbol{v}_{c / d_{2}}^{d_{2}}\right)^{T} A_{d_{1}}^{d_{2}}\left(\mathbf{b}_{c / d_{1}}^{d_{1}}+\boldsymbol{v}_{c / d_{1}}^{d_{1}}\right)
\end{aligned}
$$

Similar to the vector LOS analysis, Eq. (42) can be expanded and solved for the measured angle. The result of this is given by

$$
\begin{gathered}
\mathbf{b}_{c / d_{2}}^{c^{T}} \mathbf{b}_{c / d_{1}}^{c}=\mathbf{b}_{c / d_{2}}^{d_{2}^{T}} A_{d_{1}}^{d_{2}} \mathbf{b}_{c / d_{1}}^{d_{1}}+\mathbf{b}_{c / d_{2}}^{d_{2}^{T}} A_{d_{1}}^{d_{2}} \boldsymbol{v}_{c / d_{1}}^{d_{1}}+\boldsymbol{v}_{c / d_{2}}^{d_{2}^{T}} A_{d_{1}}^{d_{2}} \mathbf{b}_{c / d_{1}}^{d_{1}} \\
\quad+\boldsymbol{v}_{c / d_{2}}^{d_{2}^{T}} A_{d_{1}}^{d_{2}} \boldsymbol{v}_{c / d_{1}}^{d_{1}}-\mathbf{b}_{c / d_{2}}^{c^{T}} \boldsymbol{v}_{c / d_{1}}^{c}-\boldsymbol{v}_{c / d_{2}}^{c^{T}} \mathbf{b}_{c / d_{1}}^{c}-\boldsymbol{v}_{c / d_{2}}^{c^{T}} \boldsymbol{v}_{c / d_{1}}^{c}
\end{gathered}
$$

The expression in Eq. (43) can be used to determine the mean and covariance of the angle cosine, which entirely describes the probability distribution of this relationship. This methodology is again permitted by the properties assumed of the measurement noise and the linearity of the expression:

$$
\begin{gathered}
\mu_{\theta}=E\left\{\mathbf{b}_{c / d_{2}}^{c^{T}} \mathbf{b}_{c / d_{1}}^{c}\right\}=\mathbf{b}_{c / d_{2}}^{d_{2}^{T}} A_{d_{1}}^{d_{2}} \mathbf{b}_{c / d_{1}}^{d_{1}} \\
R_{\theta_{d_{2} / d_{1}}}=E\left\{\left(\mathbf{b}_{c / d_{2}}^{c^{T}} \mathbf{b}_{c / d_{1}}^{c}-\mu_{\theta}\right)^{2}\right\}
\end{gathered}
$$

To complete the expression for the angle variance, Eqs. (43) and (44) are substituted into Eq. (45) and then expanded to yield the expression given by

$$
\begin{aligned}
& R_{\theta_{d_{2} / d_{1}}}=E\left\{\mathbf{b}_{c / d_{2}}^{d_{2}^{T}} A_{d_{1}}^{d_{2}} \boldsymbol{v}_{c / d_{1}}^{d_{1}} \boldsymbol{v}_{c / d_{1}}^{d_{1}^{T}} A_{d_{1}}^{d_{2}^{T}} \mathbf{b}_{c / d_{2}}^{d_{2}}\right. \\
& +\mathbf{b}_{c / d_{2}}^{d_{2}^{T}} A_{d_{1}}^{d_{2}} \boldsymbol{v}_{c / d_{1}}^{d_{1}} \mathbf{b}_{c / d_{1}}^{d_{1}^{T}} A_{d_{1}}^{d_{2}^{T}} \boldsymbol{v}_{c / d_{2}}^{d_{2}}+\mathbf{b}_{c / d_{2}}^{d_{2}^{T}} A_{d_{1}}^{d_{2}} \boldsymbol{v}_{c / d_{1}}^{d_{1}} \boldsymbol{v}_{c / d_{1}}^{d_{1}^{T}} A_{d_{1}}^{d_{2}^{T}} \boldsymbol{v}_{c / d_{2}}^{d_{2}} \\
& -\mathbf{b}_{c / d_{2}}^{d_{2}^{T}} A_{d_{1}}^{d_{2}} \boldsymbol{v}_{c / d_{1}}^{d_{1}} \boldsymbol{v}_{c / d_{1}}^{c^{T}} \mathbf{b}_{c / d_{2}}^{c}-\mathbf{b}_{c / d_{2}}^{d_{2}^{T}} A_{d_{1}}^{d_{2}} \boldsymbol{v}_{c / d_{1}}^{d_{1}} \mathbf{b}_{c / d_{1}}^{c^{T}} \boldsymbol{v}_{c / d_{2}}^{c} \\
& -\mathbf{b}_{c / d_{2}}^{d_{2}^{T}} A_{d_{1}}^{d_{2}} \boldsymbol{v}_{c / d_{1}}^{d_{1}} \boldsymbol{v}_{c / d_{1}}^{c^{T}} \boldsymbol{v}_{c / d_{2}}^{c}+\boldsymbol{v}_{c / d_{2}}^{d_{2}^{T}} A_{d_{1}}^{d_{2}} \mathbf{b}_{c / d_{1}}^{d_{1}} \boldsymbol{v}_{c / d_{1}}^{d_{1}^{T}} A_{d_{1}}^{d_{2}^{T}} \mathbf{b}_{c / d_{2}}^{d_{2}} \\
& +\boldsymbol{v}_{c / d_{2}}^{d_{2}^{T}} A_{d_{1}}^{d_{2}} \mathbf{b}_{c / d_{1}}^{d_{1}} \mathbf{b}_{c / d_{1}}^{d_{1}^{T}} A_{d_{1}}^{d_{2}^{T}} \boldsymbol{v}_{c / d_{2}}^{d_{2}}+\boldsymbol{v}_{c / d_{2}}^{d_{2}^{T}} A_{d_{1}}^{d_{2}} \mathbf{b}_{c / d_{1}}^{d_{1}} \boldsymbol{v}_{c / d_{1}}^{d_{1}^{T}} A_{d_{1}}^{d_{2}^{T}} \boldsymbol{v}_{c / d_{2}}^{d_{2}} \\
& -\boldsymbol{v}_{c / d_{2}}^{d_{2}^{T}} A_{d_{1}}^{d_{2}} \mathbf{b}_{c / d_{1}}^{d_{1}} \boldsymbol{v}_{c / d_{1}}^{c^{T}} \mathbf{b}_{c / d_{2}}^{c}-\boldsymbol{v}_{c / d_{2}}^{d_{2}^{T}} A_{d_{1}}^{d_{2}} \mathbf{b}_{c / d_{1}}^{d_{1}} \mathbf{b}_{c / d_{1}}^{c^{T}} \boldsymbol{v}_{c / d_{2}}^{c} \\
& -\boldsymbol{v}_{c / d_{2}}^{d_{2}^{T}} A_{d_{1}}^{d_{2}} \mathbf{b}_{c / d_{1}}^{d_{1}} \boldsymbol{v}_{c / d_{1}}^{c^{T}} \boldsymbol{v}_{c / d_{2}}^{c}+\boldsymbol{v}_{c / d_{2}}^{d_{2}^{T}} A_{d_{1}}^{d_{2}} \boldsymbol{v}_{c / d_{1}}^{d_{1}} \boldsymbol{v}_{c / d_{1}}^{d_{1}^{T}} A_{d_{1}}^{d_{2}^{T}} \mathbf{b}_{c / d_{2}}^{d_{2}} \\
& +\boldsymbol{v}_{c / d_{2}}^{d_{2}^{T}} A_{d_{1}}^{d_{2}} \boldsymbol{v}_{c / d_{1}}^{d_{1}} \mathbf{b}_{c / d_{1}}^{d_{1}^{T}} A_{d_{1}}^{d_{2}^{T}} \boldsymbol{v}_{c / d_{2}}^{d_{2}}+\boldsymbol{v}_{c / d_{2}}^{d_{2}^{T}} A_{d_{1}}^{d_{2}} \boldsymbol{v}_{c / d_{1}}^{d_{1}} \boldsymbol{v}_{c / d_{1}}^{d_{1}^{T}} A_{d_{1}}^{d_{2}^{T}} \boldsymbol{v}_{c / d_{2}}^{d_{2}} \\
& -\boldsymbol{v}_{c / d_{2}}^{d_{2}^{T}} A_{d_{1}}^{d_{2}} \boldsymbol{v}_{c / d_{1}}^{d_{1}} \boldsymbol{v}_{c / d_{1}}^{c^{T}} \mathbf{b}_{c / d_{2}}^{c}-\boldsymbol{v}_{c / d_{2}}^{d_{2}^{T}} A_{d_{1}}^{d_{2}} \boldsymbol{v}_{c / d_{1}}^{d_{1}} \mathbf{b}_{c / d_{1}}^{c^{T}} \boldsymbol{v}_{c / d_{2}}^{c} \\
& -\boldsymbol{v}_{c / d_{2}}^{d_{2}^{T}} A_{d_{1}}^{d_{2}} \boldsymbol{v}_{c / d_{1}}^{d_{1}} \boldsymbol{v}_{c / d_{1}}^{c^{T}} \boldsymbol{v}_{c / d_{2}}^{c}-\mathbf{b}_{c / d_{2}}^{c^{T}} \boldsymbol{v}_{c / d_{1}}^{c} \boldsymbol{v}_{c / d_{1}}^{d_{1}^{T}} A_{d_{1}}^{d_{2}^{T}} \mathbf{b}_{c / d_{1}}^{d_{1}} \\
& -\mathbf{b}_{c / d_{2}}^{c^{T}} \boldsymbol{v}_{c / d_{1}}^{c} \mathbf{b}_{c / d_{1}}^{d_{1}^{T}} A_{d_{1}}^{d_{2}^{T}} \boldsymbol{v}_{c / d_{2}}^{d_{2}}-\mathbf{b}_{c / d_{2}}^{c^{T}} \boldsymbol{v}_{c / d_{1}}^{c} \boldsymbol{v}_{c / d_{1}}^{d_{1}^{T}} A_{d_{1}}^{d_{2}^{T}} \boldsymbol{v}_{c / d_{2}}^{d_{2}} \\
& +\mathbf{b}_{c / d_{2}}^{c^{T}} \boldsymbol{v}_{c / d_{1}}^{c} \boldsymbol{v}_{c / d_{1}}^{c^{T}} \mathbf{b}_{c / d_{2}}^{c}+\mathbf{b}_{c / d_{2}}^{c^{T}} \boldsymbol{v}_{c / d_{1}}^{c} \mathbf{b}_{c / d_{1}}^{c^{T}} \boldsymbol{v}_{c / d_{2}}^{c} \\
& +\mathbf{b}_{c / d_{2}}^{c^{T}} \boldsymbol{v}_{c / d_{1}}^{c} \boldsymbol{v}_{c / d_{1}}^{c^{T}} \boldsymbol{v}_{c / d_{2}}^{c}-\boldsymbol{v}_{c / d_{2}}^{c^{T}} \mathbf{b}_{c / d_{1}}^{c} \boldsymbol{v}_{c / d_{1}}^{d_{1}^{T}} A_{d_{1}}^{d_{2}^{T}} \mathbf{b}_{c / d_{2}}^{d_{2}} \\
& -\boldsymbol{v}_{c / d_{2}}^{c^{T}} \mathbf{b}_{c / d_{1}}^{c} \mathbf{b}_{c / d_{1}}^{d_{1}^{T}} A_{d_{1}}^{d_{2}^{T}} \boldsymbol{v}_{c / d_{2}}^{d_{2}}-\boldsymbol{v}_{c / d_{2}}^{c^{T}} \mathbf{b}_{c / d_{1}}^{c} \boldsymbol{v}_{c / d_{1}}^{d_{1}^{T}} A_{d_{1}}^{d_{2}^{T}} \boldsymbol{v}_{c / d_{2}}^{d_{2}} \\
& +\boldsymbol{v}_{c / d_{2}}^{c^{T}} \mathbf{b}_{c / d_{1}}^{c} \boldsymbol{v}_{c / d_{1}}^{c^{T}} \mathbf{b}_{c / d_{2}}^{c}+\boldsymbol{v}_{c / d_{2}}^{c^{T}} \mathbf{b}_{c / d_{1}}^{c} \mathbf{b}_{c / d_{1}}^{c^{T}} \boldsymbol{v}_{c / d_{2}}^{c} \\
& +\boldsymbol{v}_{c / d_{2}}^{c^{T}} \mathbf{b}_{c / d_{1}}^{c} \boldsymbol{v}_{c / d_{1}}^{c^{T}} \boldsymbol{v}_{c / d_{2}}^{c}-\boldsymbol{v}_{c / d_{2}}^{c^{T}} \boldsymbol{v}_{c / d_{1}}^{c} \boldsymbol{v}_{c / d_{1}}^{d_{1}^{T}} A_{d_{1}}^{d_{2}^{T}} \mathbf{b}_{c / d_{2}}^{d_{2}} \\
& -\boldsymbol{v}_{c / d_{2}}^{c^{T}} \boldsymbol{v}_{c / d_{1}}^{c} \mathbf{b}_{c / d_{1}}^{d_{1}^{T}} A_{d_{1}}^{d_{2}^{T}} \boldsymbol{v}_{c / d_{2}}^{d_{2}}-\boldsymbol{v}_{c / d_{2}}^{c^{T}} \boldsymbol{v}_{c / d_{1}}^{c} \boldsymbol{v}_{c / d_{1}}^{d_{1}^{T}} A_{d_{1}}^{d_{2}^{T}} \boldsymbol{v}_{c / d_{2}}^{d_{2}} \\
& +\boldsymbol{v}_{c / d_{2}}^{c^{T}} \boldsymbol{v}_{c / d_{1}}^{c} \boldsymbol{v}_{c / d_{1}}^{c^{T}} \mathbf{b}_{c / d_{2}}^{c}+\boldsymbol{v}_{c / d_{2}}^{c^{T}} \boldsymbol{v}_{c / d_{1}}^{c} \mathbf{b}_{c / d_{1}}^{c^{T}} \boldsymbol{v}_{c / d_{2}}^{c} \\
& \left.+\boldsymbol{v}_{c / d_{2}}^{c^{T}} \boldsymbol{v}_{c / d_{1}}^{c} \boldsymbol{v}_{c / d_{1}}^{c^{T}} \boldsymbol{v}_{c / d_{2}}^{c}\right\}
\end{aligned}
$$

Fortunately, some valid simplifications exist that decrease the complexity of the variance. First, as with the LOS analysis, it has been assumed that all the noise processes are uncorrelated. As a result, all the terms with products of unlike noise components are zero. Additionally, third moments of the given noise processes are also zero due to parity (this would parallel the integral of an odd function over an even interval). After the cancellation of these terms, the variance is given by

$$
\begin{aligned}
& R_{\theta_{d_{2} / d_{1}}}=E\left\{\mathbf{b}_{c / d_{2}}^{d_{2}^{T}} A_{d_{1}}^{d_{2}} \boldsymbol{v}_{c / d_{1}}^{d_{1}} \boldsymbol{v}_{c / d_{1}}^{d_{1}^{T}} A_{d_{1}}^{d_{2}^{T}} \mathbf{b}_{c / d_{2}}^{d_{2}}\right. \\
& \quad+\boldsymbol{v}_{c / d_{2}}^{d_{2}^{T}} A_{d_{1}}^{d_{2}} \mathbf{b}_{c / d_{1}}^{d_{1}} \mathbf{b}_{c / d_{1}}^{d_{1}^{T}} A_{d_{1}}^{d_{2}^{T}} \boldsymbol{v}_{c / d_{2}}^{d_{2}}+\boldsymbol{v}_{c / d_{2}}^{c^{T}} \boldsymbol{v}_{c / d_{1}}^{c} \boldsymbol{v}_{c / d_{1}}^{c^{T}} \boldsymbol{v}_{c / d_{2}}^{c} \\
& \quad+\boldsymbol{v}_{c / d_{2}}^{d_{2}^{T}} A_{d_{1}}^{d_{2}} \boldsymbol{v}_{c / d_{1}}^{d_{1}} \boldsymbol{v}_{c / d_{1}}^{d_{1}^{T}} A_{d_{1}}^{d_{2}^{T}} \boldsymbol{v}_{c / d_{2}}^{d_{2}}+\mathbf{b}_{c / d_{2}}^{c^{T}} \boldsymbol{v}_{c / d_{1}}^{c} \boldsymbol{v}_{c / d_{1}}^{c^{T}} \mathbf{b}_{c / d_{2}}^{c} \\
& \left.\quad+\boldsymbol{v}_{c / d_{2}}^{c^{T}} \mathbf{b}_{c / d_{1}}^{c} \mathbf{b}_{c / d_{1}}^{c^{T}} \boldsymbol{v}_{c / d_{2}}^{c}\right\}
\end{aligned}
$$

Evaluation of the expectation in Eq. (47) requires that most of the terms be examined individually (as permitted by the linearity of the expectation over summation). The resulting components from the first and fifth terms are immediately acquired by factoring out the deterministic quantities from the expectation:

$$
E\left\{\mathbf{b}_{c / d_{2}}^{d_{2}^{T}} A_{d_{1}}^{d_{2}} \boldsymbol{v}_{c / d_{1}}^{d_{1}} \boldsymbol{v}_{c / d_{1}}^{d_{1}^{T}} A_{d_{1}}^{d_{2}^{T}} \mathbf{b}_{c / d_{2}}^{d_{2}}\right\}=\mathbf{b}_{c / d_{2}}^{d_{2}^{T}} A_{d_{1}}^{d_{2}} \Omega_{c / d_{1}}^{d_{1}} A_{d_{1}}^{d_{2}^{T}} \mathbf{b}_{c / d_{2}}^{d_{2}}
$$




$$
E\left\{\mathbf{b}_{c / d_{2}}^{c^{T}} \boldsymbol{v}_{c / d_{1}}^{c} \boldsymbol{v}_{c / d_{1}}^{c^{T}} \mathbf{b}_{c / d_{2}}^{c}\right\}=\mathbf{b}_{c / d_{2}}^{c^{T}} \Omega_{c / d_{2}}^{d_{2}} \mathbf{b}_{c / d_{2}}^{c}
$$

It is also helpful to note the property that the inner product is equal to the trace of its outer product counterpart, given mathematically as $\mathbf{a}^{T} \mathbf{b}=\operatorname{tr}\left(\mathbf{b a}^{T}\right)$. This can be applied to the second and sixth terms of the variance. By grouping vector quantities, we note that the following equalities are true:

$$
\begin{gathered}
\boldsymbol{v}_{c / d_{2}}^{d_{2}^{T}} A_{d_{1}}^{d_{2}} \mathbf{b}_{c / d_{1}}^{d_{1}} \mathbf{b}_{c / d_{1}}^{d_{1}^{T}} A_{d_{1}}^{d_{2}^{T}} \boldsymbol{v}_{c / d_{2}}^{d_{2}}=\left(\boldsymbol{v}_{c / d_{2}}^{d_{2}^{T}} A_{d_{1}}^{d_{2}} \mathbf{b}_{c / d_{1}}^{d_{1}} \mathbf{b}_{c / d_{1}}^{d_{1}^{T}}\right)\left(A_{d_{1}}^{d_{2}^{T}} \boldsymbol{v}_{c / d_{2}}^{d_{2}}\right) \\
=\operatorname{tr}\left\{\left(A_{d_{1}}^{d_{2}^{T}} \boldsymbol{v}_{c / d_{2}}^{d_{2}}\right)\left(\boldsymbol{v}_{c / d_{2}}^{d_{2}^{T}} A_{d_{1}}^{d_{2}} \mathbf{b}_{c / d_{1}}^{d_{1}} \mathbf{b}_{c / d_{1}}^{d_{1}^{T}}\right)\right\} \\
\boldsymbol{v}_{c / d_{2}}^{c^{T}} \mathbf{b}_{c / d_{1}}^{c} \mathbf{b}_{c / d_{1}}^{c^{T}} \boldsymbol{v}_{c / d_{2}}^{c}=\operatorname{tr}\left\{\left(\mathbf{b}_{c / d_{1}}^{c^{T}} \boldsymbol{v}_{c / d_{2}}^{c}\right)\left(\boldsymbol{v}_{c / d_{2}}^{c^{T}} \mathbf{b}_{c / d_{1}}^{c}\right)\right\}
\end{gathered}
$$

The expectation operator can be carried inside the trace functional in Eqs. (49) and (50). The third and fourth terms of Eq. (47) require an additional step. These terms are first factored into their trace counterparts:

$$
\begin{gathered}
\boldsymbol{v}_{c / d_{2}}^{c^{T}} \boldsymbol{v}_{c / d_{1}}^{c} \boldsymbol{v}_{c / d_{1}}^{c^{T}} \boldsymbol{v}_{c / d_{2}}^{c}=\operatorname{tr}\left\{\left(\boldsymbol{v}_{c / d_{1}}^{c} \boldsymbol{v}_{c / d_{1}}^{c^{T}} \boldsymbol{v}_{c / d_{2}}^{c}\right)\left(\boldsymbol{v}_{c / d_{2}}^{c^{T}}\right)\right\} \\
\boldsymbol{v}_{c / d_{2}}^{d_{2}^{T}} A_{d_{1}}^{d_{2}} \boldsymbol{v}_{c / d_{1}}^{d_{1}} \boldsymbol{v}_{c / d_{1}}^{d_{1}^{T}} A_{d_{1}}^{d_{2}^{T}} \boldsymbol{v}_{c / d_{2}}^{d_{2}} \\
=\operatorname{tr}\left\{\left(\boldsymbol{v}_{c / d_{1}}^{d_{1}} \boldsymbol{v}_{c / d_{1}}^{d_{1}^{T}} A_{d_{1}}^{d_{2}^{T}} \boldsymbol{v}_{c / d_{2}}^{d_{2}}\right)\left(\boldsymbol{v}_{c / d_{2}}^{d_{2}^{T}} A_{d_{1}}^{d_{2}}\right)\right\}
\end{gathered}
$$

Equations (51) and (52) are different from the previous terms dealt with because they involve second moments of two different random variables. We recall that given two random variables $x_{1}$ and $x_{2}$, under the assumption that they are zero mean and mutually independent, the expectation of their product squares is given as $E\left\{x_{1}^{2} x_{2}^{2}\right\}=$ $E\left\{x_{1}^{2}\right\} E\left\{x_{2}^{2}\right\}$. Applying this property to the remaining terms and collecting the previous results leads to the angle scalar variance:

$$
\begin{aligned}
& R_{\theta_{d_{2} / d_{1}}}=\operatorname{tr}\left(\mathbf{b}_{c / d_{2}}^{d_{2}} \mathbf{b}_{c / d_{2}}^{d_{2}^{T}} A_{d_{1}}^{d_{2}} \Omega_{c / d_{1}}^{d_{1}} A_{d_{1}}^{d^{T}}\right)+\operatorname{tr}\left(\mathbf{b}_{c / d_{1}}^{d_{1}} \mathbf{b}_{c / d_{1}}^{d_{1}^{T}} A_{d_{1}}^{d_{2}^{T}} \Omega_{c / d_{2}}^{d_{2}} A_{d_{1}}^{d_{2}}\right) \\
& +\operatorname{tr}\left(A_{d_{1}}^{d_{2}} \Omega_{c / d_{1}}^{d_{1}} A_{d_{1}}^{d_{2}^{T}} \Omega_{c / d_{2}}^{d_{2}}\right)+\operatorname{tr}\left(\mathbf{b}_{c / d_{2}}^{c} \mathbf{b}_{c / d_{2}}^{c^{T}} \Omega_{c / d_{1}}^{c}\right) \\
& +\operatorname{tr}\left(\mathbf{b}_{c / d_{1}}^{c} \mathbf{b}_{c / d_{1}}^{c^{T}} \Omega_{c / d_{2}}^{c}\right)+\operatorname{tr}\left(\Omega_{c / d_{1}}^{c} \Omega_{c / d_{2}}^{c}\right)
\end{aligned}
$$

If the approximation used to obtain Eq. (41) is valid for all covariance expressions in Eq. (53), then the angle cosine variance can be simplified and be determined by known quantities from the LOS observations. Noting the property $\operatorname{tr}\left(\mathbf{b a}^{T} A\right)=\mathbf{a}^{T} A \mathbf{b}$, where $A$ is a square matrix, and the fact that an attitude matrix can be split into two different matrices as $A_{d_{1}}^{d_{2}}=A_{c}^{d_{2}} A_{c}^{d_{1}^{T}}$, then the first term on the right-hand side of Eq. (3) can be simplified to

$$
\begin{aligned}
& \operatorname{tr}\left(\mathbf{b}_{c / d_{2}}^{d_{2}} \mathbf{b}_{c / d_{2}}^{d_{2}^{T}} A_{d_{1}}^{d_{2}} \Omega_{c / d_{1}}^{d_{1}} A_{d_{1}}^{d_{2}^{T}}\right)=\mathbf{b}_{c / d_{2}}^{d_{2}^{T}} A_{c}^{d_{2}} A_{d_{1}}^{c} \Omega_{c / d_{1}}^{d_{1}} A_{d_{1}}^{c^{T}} A_{c}^{d_{2}^{T}} \mathbf{b}_{c / d_{2}}^{d_{2}} \\
& \quad=\mathbf{b}_{c / d_{2}}^{d_{2}^{T}} A_{c}^{d_{2}} \Omega_{c / d_{1}}^{c} A_{c}^{d_{2}^{T}} \mathbf{b}_{c / d_{2}}^{d_{2}}=\left(A_{d_{2}}^{c} \mathbf{b}_{c / d_{2}}^{d_{2}}\right)^{T} \Omega_{c / d_{1}}^{c} A_{d_{2}}^{c} \mathbf{b}_{c / d_{2}}^{d_{2}} \\
& \quad=\mathbf{b}_{c / d_{2}}^{c^{T}} \Omega_{c / d_{1}}^{c} \mathbf{b}_{c / d_{2}}^{c}
\end{aligned}
$$

The second term can be simplified using the same method:

$$
\operatorname{tr}\left(\mathbf{b}_{c / d_{1}}^{d_{1}} \mathbf{b}_{c / d_{1}}^{d_{1}^{T}} A_{d_{1}}^{d_{2}^{T}} \Omega_{c / d_{2}}^{d_{2}} A_{d_{1}}^{d_{2}}\right)=\mathbf{b}_{c / d_{1}}^{c^{T}} \Omega_{c / d_{2}}^{c} \mathbf{b}_{c / d_{1}}^{c}
$$

Using the cyclic property of the trace, the third term can be modified as

$$
\begin{aligned}
& \operatorname{tr}\left(A_{d}^{d_{2}} \Omega_{c / d_{1}}^{d_{1}} A_{d_{1}}^{d_{2}^{T}} \Omega_{c / d_{2}}^{d_{2}}\right)=\operatorname{tr}\left(A_{c}^{d_{2}} A_{d_{1}}^{c} \Omega_{c / d_{1}}^{d_{1}} A_{d_{1}}^{c^{T}} A_{c}^{d_{2}^{T}} \Omega_{c / d_{2}}^{d_{2}}\right) \\
& \quad=\operatorname{tr}\left(A_{c}^{d_{2}} \Omega_{c / d_{1}}^{c} A_{c}^{d_{2}^{T}} \Omega_{c / d_{2}}^{d_{2}}\right)=\operatorname{tr}\left(\Omega_{c / d_{1}}^{c} A_{c}^{d_{2}^{T}} \Omega_{c / d_{2}}^{d_{2}} A_{c}^{d_{2}}\right) \\
& \quad=\operatorname{tr}\left(\Omega_{c / d_{1}}^{c} \Omega_{c / d_{2}}^{c}\right)
\end{aligned}
$$

The fourth and the fifth terms are, respectively, identical to the first and the second terms, and the last term is the same as the third term. Thus, the angle cosine variance becomes

$$
R_{\theta_{d_{2} / d_{1}}}=2\left[\mathbf{b}_{c / d_{2}}^{c^{T}} \Omega_{c / d_{1}}^{c} \mathbf{b}_{c / d_{2}}^{c}+\mathbf{b}_{c / d_{1}}^{c^{T}} \Omega_{c / d_{2}}^{c} \mathbf{b}_{c / d_{1}}^{c}+\operatorname{tr}\left(\Omega_{c / d_{1}}^{c} \Omega_{c / d_{2}}^{c}\right)\right]
$$

which is not a function of the attitude matrix $A_{d_{1}}^{d_{2}}$.

\section{B. Attitude Estimate Covariance}

With the uncertainty of all the LOS measurements characterized within $R_{\theta}$ and $R_{d_{2} / d_{1}}^{d_{2}}$, a theoretical bound can be found for the relative attitude estimate error. As described earlier, a Gaussian distribution requires only the mean and (co)variance to describe it. In the current case, the mean is zero and expressions for the (co)variances have been determined. We now seek a characterization of $P$, the covariance for the attitude-angle errors $\delta \alpha$.

Characterization of the attitude-angle-error covariance is accomplished using the Cramèr-Rao inequality. A theoretical lower bound for the covariance can be found using the Fisher information matrix $F$. The estimate covariance $P$ is bounded by the following relationship:

$$
P=E\left\{(\hat{\mathbf{x}}-\mathbf{x})(\hat{\mathbf{x}}-\mathbf{x})^{T}\right\} \geq F^{-1}
$$

where $\mathbf{x}$ is the truth, $\hat{\mathbf{x}}$ is its corresponding estimate, and the Fisher information matrix is given by

$$
F=-E\left\{\frac{\partial^{2}}{\partial \mathbf{x} \partial \mathbf{x}^{T}} \ln L(\tilde{\mathbf{y}} ; \mathbf{x})\right\}
$$

where $L(\tilde{\mathbf{y}} ; \mathbf{x})$ is the likelihood function for a measurement $\tilde{\mathbf{y}}$. Clearly, to bound the estimate covariance, all that is needed is the second derivative of the negative log-likelihood function constructed using vector measurements with their theoretical covariance expressions previously calculated. For a discussion on how $\mathbf{x}$ is related to the attitude matrix, see [25].

The uncertainty of all the measurements has been captured in the following measurements:

$$
\tilde{\mathbf{y}}=\left[\begin{array}{c}
\tilde{\mathbf{b}}_{d_{2} / d_{1}}^{d_{2}} \\
\tilde{\mathbf{b}}_{c / d_{2}}^{c^{T}} \tilde{\mathbf{b}}_{c / d_{1}}^{c}
\end{array}\right]
$$

The covariance and variance for the LOS and angle measurement, given, respectively, by $R_{d_{2} / d_{1}}^{d_{2}}$ and $R_{\theta}$, have been determined in Eqs. (40) and (53) and are restated as follows:

$$
\begin{gathered}
\tilde{\mathbf{b}}_{d_{2} / d_{1}}^{d_{2}} \sim \mathcal{N}\left(\mathbf{b}_{d_{2} / d_{1}}^{d_{2}}, R_{d_{2} / d_{1}}^{d_{2}}\right) \\
\tilde{\mathbf{b}}_{c / d_{2}}^{c^{T}} \tilde{\mathbf{b}}_{c / d_{1}}^{c} \sim \mathcal{N}\left(\mathbf{b}_{c / d_{2}}^{c^{T}} \mathbf{b}_{c / d_{1}}^{c}, R_{\theta_{d_{2} / d_{1}}}\right)
\end{gathered}
$$

Using both the measurements from Eq. (60), those taken as deterministic quantities, and the known probability density functions described by Eq. (61), a negative log-likelihood function can be constructed (neglecting terms independent of the attitude):

$$
\begin{aligned}
& J\left(A_{d_{1}}^{d_{2}}\right)=\frac{1}{2}\left(\tilde{\mathbf{b}}_{c / d_{2}}^{c^{T}} \tilde{\mathbf{b}}_{c / d_{1}}^{c}-\tilde{\mathbf{b}}_{c / d_{2}}^{d_{2}^{T}} A_{d_{1}}^{d_{2}} \tilde{\mathbf{b}}_{c / d_{1}}^{d_{1}}\right)^{2} R_{\theta_{d_{2} / d_{1}}^{-1}}^{-} \\
& \quad+\frac{1}{2}\left(\tilde{\mathbf{b}}_{d_{2} / d_{1}}^{d_{2}}-A_{d_{1}}^{d_{2}} \tilde{\mathbf{b}}_{d_{2} / d_{1}}^{d_{1}}\right)^{T} R_{d_{2} / d_{1}}^{d_{2}^{-1}}\left(\tilde{\mathbf{b}}_{d_{2} / d_{1}}^{d_{2}}-A_{d_{1}}^{d_{2}} \tilde{\mathbf{b}}_{d_{2} / d_{1}}^{d_{1}}\right)
\end{aligned}
$$

Because the attitude error is not expected to be large, a small-errorangle assumption is made in Eq. (62). The attitude can be expressed in terms of the true attitude and the angle errors $\delta \alpha$, understood here to map $\mathcal{D}_{1}$ true to $\mathcal{D}_{2}[\underline{25}]$ :

$$
A_{d_{1}}^{d_{2}}=\left(I_{3 \times 3}-\left[\delta \boldsymbol{\alpha}_{d_{1}}^{d_{2}} \times\right]\right) A_{d_{1} \text { true }}^{d_{2}}
$$

Substituting Eq. (3) into Eq. (2) and taking the appropriate partials 
with respect to $\delta \boldsymbol{\alpha}_{d_{1}}^{d_{2}}$ leads to the following covariance:

$$
\begin{aligned}
P_{d_{1}}^{d_{2}} & \equiv E\left\{\delta \boldsymbol{\alpha}_{d_{1}}^{d_{2}} \delta \boldsymbol{\alpha}_{d_{1}}^{d_{2}^{T}}\right\} \\
& =\left(\left[A_{d_{1}}^{d_{2}} \mathbf{b}_{c / d_{1}}^{d_{1}} \times\right] \mathbf{b}_{c / d_{2}}^{d_{2}} R_{\theta_{d_{2} / d_{1}}}^{-1} \mathbf{b}_{c / d_{2}}^{d_{2}^{T}}\left[A_{d_{1}}^{d_{2}} \mathbf{b}_{c / d_{1}}^{d_{1}} \times\right]^{T}\right. \\
& \left.+\left[A_{d_{1}}^{d_{2}} \mathbf{b}_{d_{2} / d_{1}}^{d_{1}} \times\right] R_{d_{2} / d_{1}}^{d_{1}^{-1}}\left[A_{d_{1}}^{d_{2}} \mathbf{b}_{d_{2} / d_{1}}^{d_{1}} \times\right]^{T}\right)^{-1}
\end{aligned}
$$

where the subscript true has been removed because it is understood that this matrix is evaluated using the true variables (see [25] for more details). Note that $R_{d_{2} / d_{1}}^{d_{2}}$, given by Eq. (41), is a singular matrix. As shown before, this matrix can be effectively replaced by

$$
R_{d_{2} / d_{1}}^{d_{2}}+\frac{1}{2} \mathbf{b}_{d_{2} / d_{1}}^{d_{2}} \mathbf{b}_{d_{2} / d_{1}}^{d_{2}^{T}} \operatorname{tr}\left(R_{d_{2} / d_{1}}^{d_{2}}\right)
$$

which is a nonsingular matrix. The estimated attitude matrix must be used in Eq. (64) to compute the covariance. Also, the true values for the $\mathbf{b}$ vectors can effectively be replaced with their respective measured or estimated values, which leads to only second-order error effects, as stated previously.

A discussion on the observability of the three-vehicle configuration is now given. The Fisher information matrix $F_{d_{1}}^{d_{2}}$ is given by the inverse of $P_{d_{1}}^{d_{2}}$. Using the attitude mappings in Eq. (64), this matrix can be written as

$$
\begin{aligned}
& F_{d_{1}}^{d_{2}}=\left[\mathbf{b}_{c / d_{1}}^{d_{2}} \times\right] \mathbf{b}_{c / d_{2}}^{d_{2}} R_{\theta_{d_{2} / d_{1}}}^{-1} \mathbf{b}_{c / d_{2}}^{d_{2}^{T}}\left[\mathbf{b}_{c / d_{1}}^{d_{2}} \times\right]^{T} \\
& +\left[\mathbf{b}_{d_{2} / d_{1}}^{d_{2}} \times\right] R_{d_{2} / d_{1}}^{d_{1}^{-1}}\left[\mathbf{b}_{d_{2} / d_{1}}^{d_{2}} \times\right]^{T}
\end{aligned}
$$

We now show that using planar vectors yields an unobservable system. For this case, one vector can be represented by a linear combination of the other vectors, and so, for example,

$$
\mathbf{b}_{c / d_{1}}^{d_{2}}=c_{1} \mathbf{b}_{c / d_{2}}^{d_{2}}+c_{2} \mathbf{b}_{d_{2} / d_{1}}^{d_{2}}
$$

with arbitrary $c_{1}$ and $c_{2}$ in $[-1,1]$. Substituting Eq. (66) into Eq. (ㄷ) yields

$$
F_{d_{1}}^{d_{2}}=\left[\mathbf{b}_{d_{2} / d_{1}}^{d_{2}} \times\right]\left(c_{2}^{2} \mathbf{b}_{c / d_{2}}^{d_{2}} R_{d_{d_{2} / d_{1}}}^{-1} \mathbf{b}_{c / d_{2}}^{d_{2}^{T}}+R_{d_{2} / d_{1}}^{d_{1}^{-1}}\right)\left[\mathbf{b}_{d_{2} / d_{1}}^{d_{2}} \times\right]^{T}
$$

Clearly, this matrix is singular, which means that using planar vectors yields an unobservable system. One of the vectors must be out of the plane formed by the other two vectors for observability. This requires that the sensor/emitter location of one vehicle is not in the same plane as that formed by the sensors of the other two vehicles.

A simulation case is now shown to assess the effects of moving a vector out of the plane formed by the first two vectors. For this simulation, $\mathbf{b}_{c / d_{1}}^{d_{2}}=\left[\begin{array}{lll}1 & 0 & 0\end{array}\right]^{T}, \mathbf{b}_{c / d_{2}}^{d_{2}}=\left[\begin{array}{lll}0 & 1 & 0\end{array}\right]^{T}$, and

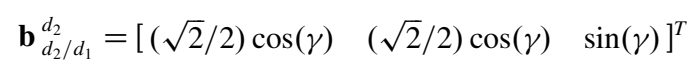

where $\gamma$ varies from near 0 deg (i.e., nearly planar vectors) to $90 \mathrm{deg}$. For simplicity, $R_{d_{2} / d_{1}}^{d_{2}}$ is assumed to be isotropic with scalar value equal to $R_{\theta_{d_{2} / d_{1}}}$, which is assumed to be $\left(17 \times 10^{-6}\right)^{2} \mathrm{rad}^{2}$. A plot of the average $3 \sigma$ bounds versus $\gamma$ is shown in Fig. $\underline{5}$. This shows that even a small movement of the vector out of the plane can provide significant improvements in attitude accuracy.

Because the analysis for the relative attitude mappings from $\mathcal{C}$ to $\mathcal{D}_{1}$ and from $\mathcal{C}$ to $\mathcal{D}_{2}$ follows similarly to the previous analysis, only the results will be given. The equations for the $\mathcal{C}$-to- $\mathcal{D}_{1}$ mapping are given by

$$
\mathbf{b}_{c / d_{1}}^{d_{1}}=A_{c}^{d_{1}} \mathbf{b}_{c / d_{1}}^{c}
$$

$$
\mathbf{b}_{d_{2} / d_{1}}^{d_{2}^{T}} \mathbf{b}_{c / d_{2}}^{d_{2}}=\left(A_{d_{1}}^{d_{2}} \mathbf{b}_{d_{2} / d_{1}}^{d_{1}}\right)^{T}\left(A_{c}^{d_{2}} \mathbf{b}_{c / d_{2}}^{c}\right)=\mathbf{b}_{d_{2} / d_{1}}^{d_{1}^{T}} A_{c}^{d_{1}} \mathbf{b}_{c / d_{2}}^{c}
$$

The LOS measurement-error covariance can be shown to be

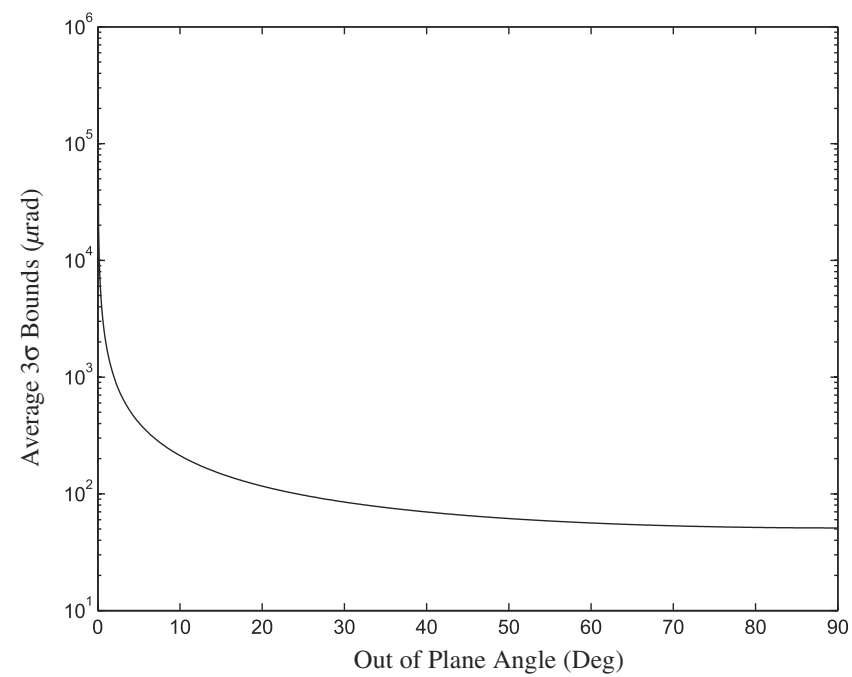

Fig. 5 Assessment of out-of-plane vector effect.

$$
R_{c / d_{1}}^{d_{1}}=A_{c}^{d_{1}} \Omega_{c / d_{1}}^{c} A_{c}^{d_{1}^{T}}+\Omega_{c / d_{1}}^{d_{1}}
$$

The variance for the angle cosine, $\mathbf{b}_{d_{2} / d_{1}}^{d_{2}^{T}} \mathbf{b}_{c / d_{2}}^{d_{2}}$, is similarly given by

$$
\begin{aligned}
& R_{\theta_{c / d_{1}}}=\operatorname{tr}\left(\mathbf{b}_{d_{2} / d_{1}}^{d_{1}} \mathbf{b}_{d_{2} / d_{1}}^{d_{1}^{T}} A_{c}^{d_{1}} \Omega_{c / d_{2}}^{c} A_{c}^{d_{1}^{T}}\right) \\
& \quad+\operatorname{tr}\left(\mathbf{b}_{c / d_{2}}^{c^{T}} A_{c}^{d_{1}^{T}} \Omega_{d_{2} / d_{1}}^{d_{1}} A_{c}^{d_{1}} \mathbf{b}_{c / d_{2}}^{c}\right)+\operatorname{tr}\left(\Omega_{c / d_{2}}^{c} A_{c}^{d_{1}^{T}} \Omega_{d_{2} / d_{1}}^{d_{1}} A_{c}^{d_{1}}\right) \\
& \quad+\operatorname{tr}\left(\mathbf{b}_{d_{2} / d_{1}}^{d_{2}} \mathbf{b}_{d_{2} / d_{1}}^{d_{2}^{T}} \Omega_{c / d_{2}}^{d_{2}}\right)+\operatorname{tr}\left(\mathbf{b}_{c / d_{2}}^{d_{2}} \mathbf{b}_{c / d_{2}}^{d_{2}^{T}} \Omega_{d_{2} / d_{1}}^{d_{2}}\right) \\
& \quad+\operatorname{tr}\left(\Omega_{c / d_{2}}^{d_{2}} \Omega_{d_{2} / d_{1}}^{d_{2}}\right)
\end{aligned}
$$

which can be simplified to

$$
\begin{aligned}
& R_{\theta_{c / d_{1}}}=2\left\{\mathbf{b}_{d_{2} / d_{1}}^{d_{2}^{T}} \Omega_{c / d_{2}}^{d_{2}} \mathbf{b}_{c / d_{2}}^{d_{2}}+\mathbf{b}_{c / d_{2}}^{d_{2}^{T}} \Omega_{d_{2} / d_{1}}^{d_{2}} \mathbf{b}_{c / d_{2}}^{d_{2}}\right. \\
& \left.\quad+\operatorname{tr}\left(\Omega_{c / d_{2}}^{d_{2}} \Omega_{d_{2} / d_{1}}^{d_{2}}\right)\right\}
\end{aligned}
$$

Constructing the appropriate negative log-likelihood function counterpart to Eq. (62) and following through the same progression of simplifications will yield an analog to Eq. (64):

$$
\begin{aligned}
P_{c}^{d_{1}} & =\left(\left[A_{c}^{d_{1}} \mathbf{b}_{c / d_{2}}^{c} \times\right] \mathbf{b}_{d_{2} / d_{1}}^{d_{1}} R_{\theta_{c / d_{1}}}^{-1} \mathbf{b}_{d_{2} / d_{1}}^{d_{1}^{T}}\left[A_{c}^{d_{1}} \mathbf{b}_{c / d_{2}}^{c} \times\right]^{T}\right. \\
& \left.+\left[A_{c}^{d_{1}} \mathbf{b}_{c / d_{1}}^{c} \times\right] R_{c / d_{1}}^{d_{1}^{-1}}\left[A_{c}^{d_{1}} \mathbf{b}_{c / d_{1}}^{c} \times\right]^{T}\right)^{-1}
\end{aligned}
$$

The equations for the $\mathcal{C}$-to- $\mathcal{D}_{2}$ mapping are given by

$$
\mathbf{b}_{c / d_{2}}^{d_{2}}=A_{c}^{d_{2}} \mathbf{b}_{c / d_{2}}^{c}
$$

$$
\mathbf{b}_{d_{1} / d_{2}}^{d_{1}^{T}} \mathbf{b}_{c / d_{1}}^{d_{1}}=\left(A_{d_{2}}^{d_{1}} \mathbf{b}_{d_{1} / d_{2}}^{d_{2}}\right)^{T}\left(A_{c}^{d_{1}} \mathbf{b}_{c / d_{1}}^{c}\right)=\mathbf{b}_{d_{1} / d_{2}}^{d^{T}} A_{c}^{d_{2}} \mathbf{b}_{c / d_{1}}^{c}
$$

The LOS measurement-error covariance can be shown to be

$$
R_{c / d_{2}}^{d_{2}}=A_{c}^{d_{2}} \Omega_{c / d_{2}}^{c} A_{c}^{d_{2}^{T}}+\Omega_{c / d_{2}}^{d_{2}}
$$

The variance for the angle cosine, $\mathbf{b}_{d_{1} / d_{2}}^{d_{1}^{T}} \mathbf{b}_{c / d_{1}}^{d_{1}}$, is similarly given by 


$$
\begin{aligned}
& R_{\theta_{c / d_{2}}}=\operatorname{tr}\left(\mathbf{b}_{d_{1} / d_{2}}^{d_{2}} \mathbf{b}_{d_{1} / d_{2}}^{d_{2}^{T}} A_{c}^{d_{2}} \Omega_{c / d_{1}}^{c} A_{c}^{d_{2}^{T}}\right) \\
& \quad+\operatorname{tr}\left(\mathbf{b}_{c / d_{1}}^{c} \mathbf{b}_{c / d_{1}}^{c^{T}} A_{c}^{d_{2}^{T}} \Omega_{d_{1} / d_{2}}^{d_{2}} A_{c}^{d_{2}}\right)+\operatorname{tr}\left(\Omega_{c / d_{1}}^{c} A_{c}^{d_{2}^{T}} \Omega_{d_{1} / d_{2}}^{d_{2}} A_{c}^{d_{2}}\right) \\
& \quad+\operatorname{tr}\left(\mathbf{b}_{d_{1} / d_{2}}^{d_{1}} \mathbf{b}_{d_{1} / d_{2}}^{d_{1}^{T}} \Omega_{c / d_{1}}^{d_{1}}\right)+\operatorname{tr}\left(\mathbf{b}_{c / d_{1}}^{d_{1}} \mathbf{b}_{c / d_{1}}^{d_{1}^{T}} \Omega_{d_{1} / d_{2}}^{d_{1}}\right) \\
& \quad+\operatorname{tr}\left(\Omega_{c / d_{1}}^{d_{1}} \Omega_{d_{1} / d_{2}}^{d_{1}}\right)
\end{aligned}
$$

which can be simplified to

$$
\begin{aligned}
& R_{\theta_{c / d_{2}}}=2\left\{\mathbf{b}_{d_{1} / d_{2}}^{d_{1}^{T}} \Omega_{c / d_{1}}^{d_{1}} \mathbf{b}_{d_{1} / d_{2}}^{d_{1}}+\mathbf{b}_{c / d_{1}}^{d_{1}^{T}} \Omega_{d_{1} / d_{2}}^{d_{1}} \mathbf{b}_{c / d_{1}}^{d_{1}}\right. \\
& \left.\quad+\operatorname{tr}\left(\Omega_{c / d_{1}}^{d_{1}} \Omega_{d_{1} / d_{2}}^{d_{1}}\right)\right\}
\end{aligned}
$$

Constructing the appropriate negative log-likelihood function counterpart to Eq. (62) and following through the same progression of simplifications will yield an analog to Eq. (64):

$$
\begin{aligned}
P_{c}^{d_{2}} & =\left(\left[A_{c}^{d_{2}} \mathbf{b}_{c / d_{1}}^{c} \times\right] \mathbf{b}_{d_{1} / d_{2}}^{d_{2}} R_{\theta_{c / d_{2}}}^{-1} \mathbf{b}_{d_{1} / d_{2}}^{d_{2}^{T}}\left[A_{c}^{d_{2}} \mathbf{b}_{c / d_{1}}^{c} \times\right]^{T}\right. \\
& \left.+\left[A_{c}^{d_{2}} \mathbf{b}_{c / d_{2}}^{c} \times\right] R_{c / d_{2}}^{d^{-1}}\left[A_{c}^{d_{2}} \mathbf{b}_{c / d_{2}}^{c} \times\right]^{T}\right)^{-1}
\end{aligned}
$$

\section{Nonparallel-Beam Case}

All previous cases assume perfect alignment in the beams transmitted between the neighboring vehicles. The third case assumes nonparallel beams. The intent is to replace the LOS vectors in Sec. $\underline{\mathrm{V}}$ with the source-to-source LOS vectors, as shown in Fig. $\underline{6}$, using the range measurement and FPD-source displacement. The misalignment in the beams can arise when the FPD and the beam source do not reside in the same location, as shown in Fig. 6. To establish a common vector represented in two different coordinate frames, the displacement vectors between each beam source and respective FPD must be used for each vehicle, denoted by $\mathbf{z}_{c / c}^{c}$ for the chief and $\mathbf{z}_{d_{1} / d_{1}}^{d_{1}}$ for the deputy. These vectors are well known through calibration procedures and remain fixed with respect to the vehicle body. Because the FPD measurement gives a unit vector in the direction of the incoming beam, then range information is also needed to determine the relative position between the FPD on the receiving vehicle and the beam source of the other vehicle. This vector and the displacement between the FPD and laser on the chief frame can be used to generate a triangle. From Fig. $\underline{6}$, we have

$$
\begin{gathered}
\mathbf{y}_{c / d_{1}}^{c}=\mathbf{r}_{c / d_{1}}^{c}-\mathbf{z}_{c / c}^{c} \\
\mathbf{y}_{c / d_{1}}^{d_{1}}=\mathbf{r}_{c / d_{1}}^{d_{1}}+\mathbf{z}_{d_{1} / d_{1}}^{d_{1}}
\end{gathered}
$$

where $\mathbf{r}_{c / d_{1}}^{c}=r_{c / d_{1}}^{c} \mathbf{b}_{c / d_{1}}^{c}$ and $\mathbf{r}_{c / d_{1}}^{d_{1}}=r_{c / d_{1}}^{d_{1}} \mathbf{b}_{c / d_{1}}^{d_{1}}$. The quantities $r_{c / d_{1}}^{c} \equiv\left\|\mathbf{r}_{c / d_{1}}^{c}\right\|$ and $r_{c / d_{1}}^{d_{1}} \equiv\left\|\mathbf{r}_{c / d_{1}}^{d_{1}}\right\|$ are given from range

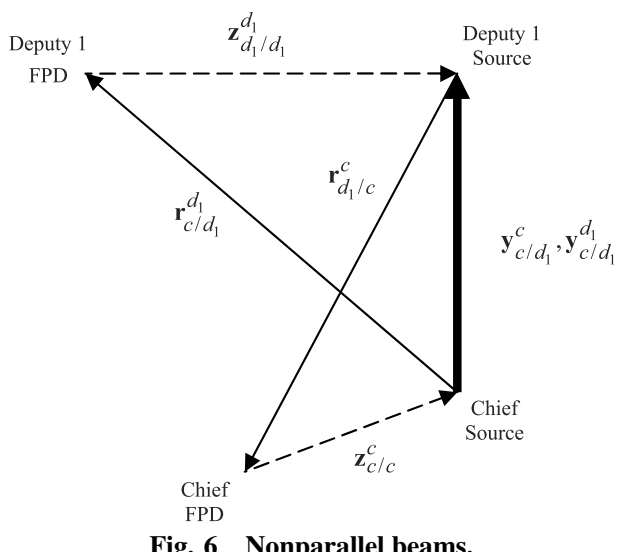

Fig. 6 Nonparallel beams. observations between vehicles. The vectors $\mathbf{y}_{c / d_{1}}^{c}$ and $\mathbf{y}_{c / d_{1}}^{d_{1}}$ are common sides of the triangle. Note that the respective measured vectors for $\mathbf{y}_{c / d_{1}}^{c}$ and $\mathbf{y}_{c / d_{1}}^{d_{1}}$ may not have the same length, due to noise. Therefore, these common vector observations are related between frames by the following attitude transformation:

$$
\overline{\mathbf{b}}_{c / d_{1}}^{c}=A_{d_{1}}^{c} \overline{\mathbf{b}}_{c / d_{1}}^{d_{1}}
$$

where $\overline{\mathbf{b}}_{c / d_{1}}^{c} \equiv \mathbf{y}_{c / d_{1}}^{c} /\left\|\mathbf{y}_{c / d_{1}}^{c}\right\|$ and $\overline{\mathbf{b}}_{c / d_{1}}^{d_{1}} \equiv \mathbf{y}_{c / d_{1}}^{d_{1}} /\left\|\mathbf{y}_{c / d_{1}}^{d_{1}}\right\|$ are unit vectors. Measurement errors exist for both the range measurements and focal-plane measurements. Ignoring subscripts and superscripts, we wish to obtain a linear measurement-error model as follows: $\tilde{\overline{\mathbf{b}}}=\overline{\mathbf{b}}+\overline{\boldsymbol{v}}$. The measured $\mathbf{y}$ vector, denoted by $\tilde{\mathbf{y}}$, can be written in terms of the measured LOS and range:

$$
\tilde{\mathbf{y}}=\left(r+v_{r}\right)(\mathbf{b}+\boldsymbol{v})+\mathbf{z}=\mathbf{y}+\mathbf{w}
$$

where the variance of the range error $v_{r}$ is denoted by $\varpi^{2}$, the covariance of $\boldsymbol{v}$ is $\Omega$ as before, and $\mathbf{w} \equiv v_{r} \mathbf{b}+r \boldsymbol{v}+v_{r} \boldsymbol{v}$. Because $v_{r}$ and $\boldsymbol{v}$ are uncorrelated, then $E\left\{\mathbf{w} \mathbf{w}^{T}\right\}=\varpi^{2} \mathbf{b} \mathbf{b}^{T}+r^{2} \Omega$. The covariance of $\overline{\boldsymbol{v}}$, denoted by $\bar{\Omega}$, can be derived using the same approach as shown in Sec. IV:

$$
\bar{\Omega}=\left(\frac{\partial \overline{\mathbf{b}}}{\partial \mathbf{y}}\right)\left(\varpi^{2} \mathbf{b} \mathbf{b}^{T}+r^{2} \Omega\right)\left(\frac{\partial \overline{\mathbf{b}}}{\partial \mathbf{y}}\right)^{T}
$$

with

$$
\frac{\partial \overline{\mathbf{b}}}{\partial \mathbf{y}}=\|\mathbf{y}\|^{-1}\left(I_{3 \times 3}-\|\mathbf{y}\|^{-2} \mathbf{y} \mathbf{y}^{T}\right)
$$

The algorithms and attitude-error covariances shown in Sec. $\underline{V}$ can now be directly applied using the appropriate $\tilde{\overline{\mathbf{b}}}$ measurement vectors and $\bar{\Omega}$ covariances.

Intuitively speaking, as the distance between vehicles becomes large, the beams become more parallel. We now study this effect on the measurement covariance by explicitly multiplying out the terms in Eq. (22) and using the QMM for $\Omega$, which leads to

$$
\begin{gathered}
\bar{\Omega}=\frac{r^{2} \sigma^{2}}{\|\mathbf{y}\|^{2}}\left[I_{3 \times 3}-\overline{\mathbf{b}} \overline{\mathbf{b}}^{T}-\left(\overline{\mathbf{b}}^{T} \mathbf{b}\right)^{2} \mathbf{b} \mathbf{b}^{T}+\left(\overline{\mathbf{b}}^{T} \mathbf{b}\right)\left(\overline{\mathbf{b}} \mathbf{b}^{T}+\mathbf{b} \overline{\mathbf{b}}^{T}\right)\right. \\
\left.-\mathbf{b b}^{T}\right]+\frac{\varpi^{2}}{\|\mathbf{y}\|^{2}}\left[\left(\overline{\mathbf{b}}^{T} \mathbf{b}\right)^{2} \mathbf{b} \mathbf{b}^{T}-\left(\overline{\mathbf{b}}^{T} \mathbf{b}\right)\left(\overline{\mathbf{b}} \mathbf{b}^{T}+\mathbf{b} \overline{\mathbf{b}}^{T}\right)+\mathbf{b} \mathbf{b}^{T}\right]
\end{gathered}
$$

where $\overline{\mathbf{b}} \equiv \mathbf{y} /\|\mathbf{y}\|$ has been used. When $r \rightarrow \infty$, then $\|\mathbf{y}\| \rightarrow r$ and $\overline{\mathbf{b}} \rightarrow$ b. Thus, Eq. ( $\underline{83}$ ) reduces down to

$$
\bar{\Omega}=\sigma^{2}\left(I_{3 \times 3}-\mathbf{b b}^{T}\right)=\Omega
$$

The preceding analysis demonstrates that as the distance between vehicles increases, the error in the measurement approaches and converges to that of the parallel case. Therefore, any additional error, induced by the range measurement in the nonparallel case, becomes less significant when the formation distance is large, as expected. However, the vectors also become more planar as the distance increases, and observability tends to slip away, as discussed previously. Therefore, a tradeoff exists between errors induced by the range measurements versus accuracy/observability issues for near-planar vectors. It should be noted that many three-vehicle systems may involve short-range distances though, for example, such as aircraft and robotic systems.

\section{Simulations}

The simulations use a static formation of three vehicles, with each vehicle having two FPDs and light source devices. The relative attitude mapping between each vehicle's body frame is determined from LOS measurements, assuming perfect alignment between beams. The formation configuration uses the following true LOS 
vectors:

$$
\begin{aligned}
\mathbf{b}_{c / d_{1}}^{c} & =\left[\begin{array}{c}
\sin (-30 \mathrm{deg}) \cos (35 \mathrm{deg}) \\
\sin (35 \mathrm{deg}) \\
\cos (30 \mathrm{deg}) \cos (35 \mathrm{deg})
\end{array}\right] \\
\mathbf{b}_{c / d_{2}}^{c} & =\left[\begin{array}{c}
\sin (30 \mathrm{deg}) \cos (25 \mathrm{deg}) \\
\sin (25 \mathrm{deg}) \\
\cos (30 \mathrm{deg}) \cos (25 \mathrm{deg})
\end{array}\right] \\
\mathbf{b}_{d_{1} / d_{2}}^{d_{1}} & =\left[\begin{array}{c}
-\cos (45 \mathrm{deg}) \cos (10 \mathrm{deg}) \\
\sin (10 \mathrm{deg}) \\
\sin (-45 \mathrm{deg}) \cos (10 \mathrm{deg})
\end{array}\right]
\end{aligned}
$$

The remaining three LOS truth vectors are determined from those listed in Eq. (24), without noise added, using the appropriate attitude transformation. For this configuration, the true relative attitudes are given by

$$
A_{c}^{d_{1}}=\left[\begin{array}{ccc}
0 & 0 & -1 \\
0 & 1 & 0 \\
1 & 0 & 0
\end{array}\right], \quad A_{c}^{d_{2}}=\left[\begin{array}{ccc}
1 & 0 & 0 \\
0 & 0 & 1 \\
0 & -1 & 0
\end{array}\right]
$$

For this test case, the angle from the plane formed by the vectors $\mathbf{b}_{c / d_{1}}^{c}$ and $\mathbf{b}_{c / d_{2}}^{c}$ to the vector $\mathbf{b}_{d_{1} / d_{2}}^{c}$ is about $22 \mathrm{deg}$. The LOS vectors are determined from the focal-plane measurements containing noise, which is described in Sec. III, with $\sigma=17 \times 10^{-6} \mathrm{rad}$ and $d=1$. Also, the focal length is assumed to be one. In practice, each vehicle must have two FPDs to ensure that the light source is acquired for any relative orientation; this will generally be required for many systems, such as laser communication devices. The letter $S$ is used to denote the sensor frame. The six matrix orthogonal transformations for their respective sensor frames, denoted by a subscript, used to orientate the FPD to the specific vehicle, denoted by the superscript, are given by

$$
\begin{aligned}
A_{s_{d_{1}}}^{c} & =\left[\begin{array}{ccc}
-0.9131 & -0.1871 & -0.3624 \\
-0.1871 & -0.5973 & 0.7799 \\
-0.3624 & 0.7799 & 0.5103
\end{array}\right] \\
A_{s_{c}}^{d_{1}} & =\left[\begin{array}{ccc}
-0.1623 & 0.0840 & 0.9832 \\
0.0840 & -0.9916 & 0.0986 \\
0.9832 & 0.0986 & 0.1539
\end{array}\right] \\
A_{s_{d_{1}}}^{d_{2}} & =\left[\begin{array}{ccc}
-0.6541 & -0.3990 & -0.6427 \\
-0.3990 & -0.5399 & 0.7412 \\
-0.6427 & 0.7412 & 0.1939
\end{array}\right] \\
A_{s_{d_{2}}}^{c} & =\left[\begin{array}{ccc}
-0.4394 & 0.5125 & 0.7378 \\
0.5125 & -0.5316 & 0.6744 \\
0.7378 & 0.6744 & -0.0290
\end{array}\right]
\end{aligned}
$$

$$
\begin{aligned}
A_{s_{c}}^{d_{1}} & =\left[\begin{array}{ccc}
-0.5232 & 0.3952 & 0.7550 \\
0.3952 & -0.6724 & 0.6259 \\
0.7550 & 0.6259 & 0.1955
\end{array}\right] \\
A_{s_{c}}^{d_{2}} & =\left[\begin{array}{ccc}
-0.9698 & -0.0721 & 0.2330 \\
-0.0721 & -0.8280 & -0.5561 \\
0.2330 & -0.5561 & 0.7978
\end{array}\right]
\end{aligned}
$$

Because each FPD has its own boresight axis, and the measurement covariance in Eq. (2) is described with respect to the boresight, individual sensor frames must be defined to generate the FPD measurements. The measurement-error covariance for each FPD is determined with respect to the corresponding sensor frames and must be rotated to the vehicle's body frame as well. The estimated attitudeerror covariance for each mapping is determined using either Eq. (64), Eq. (72), or Eq. (77). The relative attitude estimates are calculated by the method outlined in Sec. $\underline{V}$ from the measurement

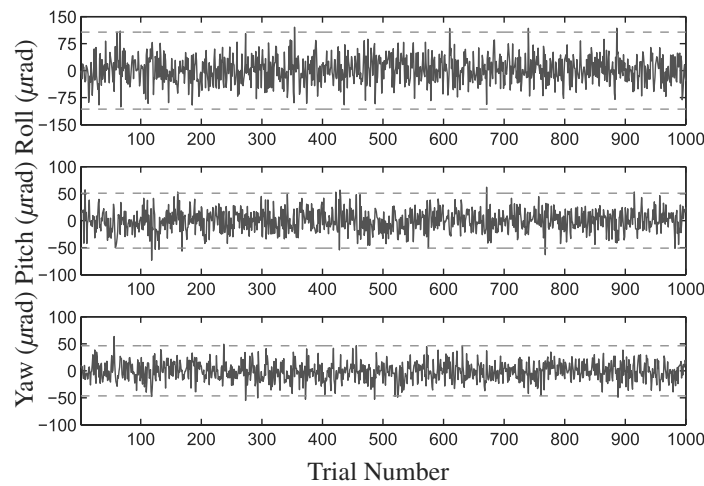

a) $\mathcal{D}_{1}$ to $\mathcal{D}_{2}$ errors

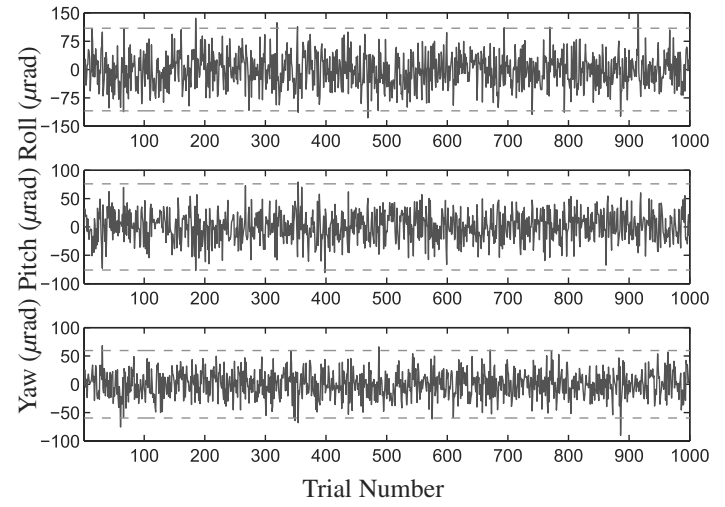

b) $\mathcal{C}$ to $\mathcal{D}_{1}$ errors

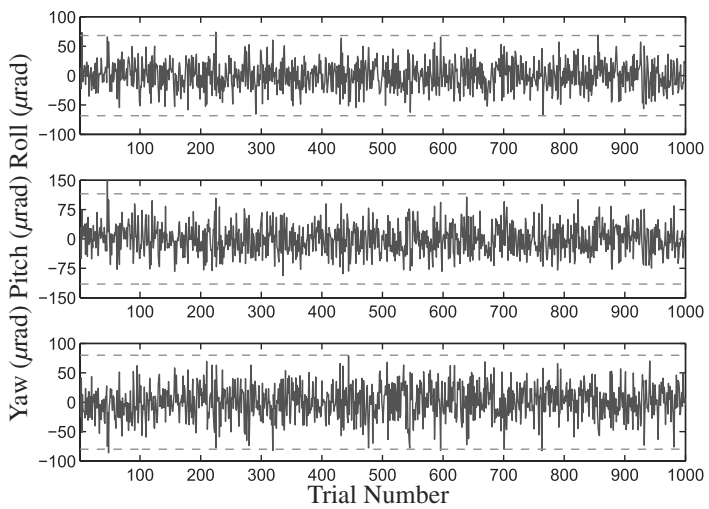

c) $\mathcal{C}$ to $\mathcal{D}_{2}$ errors

Fig. 7 Relative attitude estimate errors. 


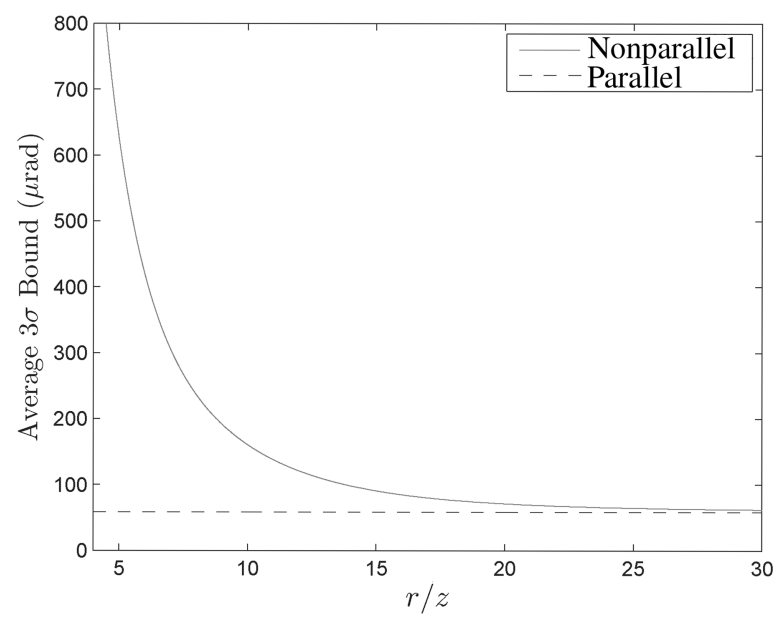

Fig. 8 Average $3 \sigma$ bounds for parallel and nonparallel cases.

containing random noise described by the measurement covariance. The configuration is considered for 1000 Monte Carlo trials. Measurements are generated in the sensor frame and rotated to the body frame to be combined with the other measurements to determine the full relative attitudes. The WFOV measurement model for the FPD LOS covariance is used. Relative attitude-angle errors are displayed with their theoretical $3 \sigma$ bounds. Figure 7 a shows the errors for the $\mathcal{D}_{1}$-to- $\mathcal{D}_{2}$ mapping. Figure $7 \mathrm{~b}$ shows the errors for the $\mathcal{C}$-to- $\mathcal{D}_{1}$ mapping, and Fig. $7 \mathrm{c}$ shows the errors for the final relative attitude mapping of $\mathcal{C}$ to $\overline{\mathcal{D}_{2}}$. Clearly, the theoretical covariance expressions provide an accurate means to predict expected performance.

A comparison of the attitude error for the nonparallel case with the parallel case as the relative distance between vehicles increases is now shown. The same three-vehicle configuration as the last simulation is considered, with each vector having the same sensor displacement, denoted by $z$. The relative distance between each vehicle is assumed to be equal, denoted by $r$. From the observation geometry shown in Fig. 6, the FPD and the range measurements made in each frame can be deduced from the true LOS vector and distance between vehicles. Range measurements are generated using a zero-mean Gaussian white noise with a standard deviation of $1 \mathrm{~m}$. FPD measurement errors are generated using the same standard deviation as before, $\sigma=17 \times 10^{-6} \mathrm{rad}$. This formation is then expanded out, incrementing all the distances between vehicles by an equal amount. The average theoretical $3 \sigma$ bounds for increasing $r / z$ are shown in Fig. 8. Clearly, as the formation size increases, for this case when $r / z$ approaches a value of 30 , the relative attitude error for the nonparallel case approaches that of the parallel case, which confirms the analysis leading to Eq. (84).

\section{Conclusions}

This paper has shown that with a three-vehicle configuration, deterministic attitude solutions are possible, assuming line-of-sight information between each vehicle pair. Covariance expressions were derived to determine the attitude-error $3 \sigma$ bounds, which closely matched with Monte Carlo simulations. Three cases were shown. One involved using the two deputy vehicles as reference points to determine the inertial attitude of the chief. Care must be taken for this case, because the attitude accuracy depends not only on the line-ofsight errors, but also on the position errors of the deputies. The second case involved using parallel beams. The advantage of this approach is that no other information, such as position-type knowledge, is required to find a solution; however, a feedback mechanism must be employed at each light source to maintain the parallel beams. Also, the closer the three vectors are to forming a plane, the worse the attitude accuracy becomes. The third case involved nonparallel beams. Additional information involving range knowledge between vehicles must be given for this case; however, the adverse effects from this range measurement become less important as the distance between the vehicles becomes larger. But, as the distance increases, the resulting vectors become more planar, which adversely affects attitude accuracy. The derived covariance expressions can be used to assess these range and planar vector effects. It is important to note that although the examples discussed in this paper primarily involve spacecraft applications, the relative attitude determination approaches shown herein can be employed to any three-vehicle system with similar sensors used here.

\section{Acknowledgments}

This work was supported through Phases I and II Small Business Technology Transfer (STTR) program grants (contract FA9453-07M-0101). The authors wish to thank Terry Tidwell from Space Photonics, Inc., and Adam M. Fosbury from the U.S. Air Force Research Laboratory Space Vehicles Directorate for their support.

\section{References}

[1] Fallon, L., "Star Sensors," Spacecraft Attitude Determination and Control, edited by J. R. Wertz, Kluwer Academic, Dordrecht, The Netherlands, 1978, pp. 184-195.

[2] Cohen, C. E., "Attitude Determination," Global Positioning System: Theory and Applications, edited by B. Parkinson, and J. Spilker, Progress in Astronautics and Aeronautics, Vol. 64, AIAA, Washington, D.C., 1996, Chap. 19.

[3] Shuster, M. D., "Deterministic Three-Axis Attitude Determination," Journal of the Astronautical Sciences, Vol. 52, No. 3, July-Sept. 2004, pp. 405-419.

[4] Wahba, G., "A Least-Squares Estimate of Satellite Attitude," SIAM Review, Vol. 7, No. 3, July 1965, p. 409. doi: $10.1137 / 1007077$

[5] Markley, F. L., and Mortari, D., "Quaternion Attitude Estimation Using Vector Observations," Journal of the Astronautical Sciences, Vol. 48, Nos. 2-3, Apr.-Sept. 2000, pp. 359-380.

[6] Cao, Y. U., Fukunaga, A. S., Kahng, A. B., and Meng, F., "Cooperative Mobile Robotics: Antecedents and Directions," Autonomous Robots, Vol. 4, No. 1, Mar. 1997, pp. 7-27. doi:10.1023/A:1008855018923

[7] Stilwell, D., and Bishop, B., "Platoons of Underwater Vehicles," IEEE Control Systems Magazine, Vol. 20, No. 6, Dec. 2000, pp. 45-52. doi:10.1109/37.887448

[8] Carpenter, J., Leitner, J., Folta, D., and Burns, R., "Benchmark Problems for Spacecraft Formation Flying Missions," AIAA Guidance, Navigation, and Control Conference, Austin, TX, AIAA Paper 20035364, Aug. 2003.

[9] DeGarmo, M., and Nelson, G., "Prospective Unmanned Aerial Vehicle Operations in the Future National Airspace System," AIAA 4th Aviation Technology, Integration and Operations (ATIO) Forum, Chicago, IL, AIAA Paper 2004-6243, Sept. 2004.

[10] Kim, S. G., Crassidis, J. L., Cheng, Y., Fosbury, A. M., and Junkins, J. L., "Kalman Filtering for Relative Spacecraft Attitude and Position Estimation," Journal of Guidance, Control, and Dynamics, Vol. 30, No. 1, Jan.-Feb. 2007, pp. 133-143. doi:10.2514/1.22377

[11] Fosbury, A. M., and Crassidis, J. L., "Relative Navigation of Air Vehicles," Journal of Guidance, Control, and Dynamics, Vol. 31, No. 4, July-Aug. 2008, pp. 824-834. doi: $10.2514 / 1.33698$

[12] Gunnam, K. K., Hughes, D. C., Junkins, J. L., and Kehtarnavaz, N., "A Vision-Based DSP Embedded Navigation Sensor," IEEE Sensors Journal, Vol. 2, No. 5, Oct. 2002, pp. 428-442. doi:10.1109/JSEN.2002.806212

[13] Sun, D., and Crassidis, J. L., "Observability Analysis of Six-Degree-ofFreedom Configuration Determination Using Vector Observations," Journal of Guidance, Control, and Dynamics, Vol. 25, No. 6, Nov.Dec. 2002, pp. 1149-1157. doi: $10.2514 / 2.4995$

[14] Spletzer, J., Das, A. K., Fierro, R., Taylor, C. J., Kumar, V., and Ostrowski, J. P., "Cooperative Localization and Control for MultiRobot Manipulation," Proceedings of the 2001 IEEE/RSJ International Conference on Intelligent Robots and Systems, Vol. 2, IEEE Publications, Piscataway, NJ, 2001, pp. 631-636.

[15] Aviv, D. G., Laser Space Communications, Artech House, Boston, 2006, Chap. 1

[16] Light, D. L., "Satellite Photogrammetry," Manual of Photogrammetry, edited by C. C. Slama, 4th ed., American Society of Photogrammetry, 
Falls Church, VA, 1980, Chap. 17.

[17] Crassidis, J. L., Cheng, Y., Fosbury, A. M., and Nebelecky, C. K., "Decentralized Attitude Estimation Using a Quaternion Covariance Intersection Approach," The F. Landis Markley Astronautics Symposium, edited by J. L. Crassidis, J. L. Junkins, K. C. Howell, Y. Oshman, and J. K. Thienel, Advances in the Astronautical Sciences, Vol. 132, Univelt, San Diego, CA, 2008, pp. 423-444.

[18] Fosbury, A. M., "Analysis of Approximations in Multi-Temporal Measurements," AIAA Guidance, Navigation, and Control Conference, Honolulu, HI, AIAA Paper 2008-7471, Aug. 2008.

[19] Shuster, M. D., "Kalman Filtering of Spacecraft Attitude and the QUEST Model," Journal of the Astronautical Sciences, Vol. 38, No. 3, July-Sept. 1990, pp. 377-393.

[20] Cheng, Y., Crassidis, J. L., and Markley, F. L., "Attitude Estimation for Large Field-of-View Sensors," Journal of the Astronautical Sciences, Vol. 54, Nos. 3-4, July-Dec. 2006, pp. 433-448.

[21] Crassidis, J. L., and Junkins, J. L., Optimal Estimation of
Dynamic Systems, Chapman \& Hall/CRC, Boca Raton, FL, 2004, p. 541.

[22] Shuster, M. D., "Constraint in Attitude Estimation Part 1: Constrained Estimation," Journal of the Astronautical Sciences, Vol. 51, No. 1, Jan.-Mar. 2003, pp. 51-74.

[23] Shuster, M. D., and Oh, S. D., "Three-Axis Attitude Determination from Vector Observations," Journal of Guidance and Control, Vol. 4, No. 1, Jan.-Feb. 1981, pp. 70-77. doi:10.2514/3.19717

[24] Crassidis, J. L., and Markley, F. L., "New Algorithm for Attitude Determination Using Global Positioning System Signals," Journal of Guidance, Control, and Dynamics, Vol. 20, No. 5, Sept.-Oct. 1997, pp. 891-896. doi: $10.2514 / 2.4162$

[25] Shuster, M. D., "Maximum Likelihood Estimation of Spacecraft Attitude," Journal of the Astronautical Sciences, Vol. 37, No. 1, Jan.Mar. 1989, pp. 79-88. 